\title{
CATÁLOGO DE LOS HALICTINI HALICTUS LATREILLE, 1804 Y LASIOGLOSSUM CURTIS, 1833 (HYMENOPTERA, APOIDEA, HALICTIDAE) DE LA PENÍNSULA IBÉRICA Y DE LAS ISLAS CANARIAS ${ }^{1}$
}

\author{
C. Ornosa*, M. López-Goñi*,F. Torres** \& D. Romero*
}

\section{RESUMEN}

C. Ornosa, M. López-Goñi, F. Torres \& D. Romero. 2013. Catálogo de los Halictini Halictus Latreille, 1804 y Lasioglossum Curtis, 1833 (Hymenoptera, Apoidea, Halictidae) de la Península lbérica y de las islas Canarias. Grael/sia, 69(2): 247-274.

En este catálogo se presenta un inventario actualizado de las especies de Halictini de los géneros Halictus Latreille, 1804 y Lasioglossum Curtis, 1833 de la Península lbérica y de las Islas Canarias. Contiene 143 especies y 161 subespecies: 126 de las especies se conocen en la fauna ibérica y 17 en las Islas Canarias y, de estas, 6 especies y 17 subespecies son endémicas, de un total de 29 subespecies presentes en las islas; 9 de las especies canarias, son comunes a ambos territorios. De todos los taxones, además de la correspondiente discusión, si procede, se incluye su lista sinonímica y su distribución geográfica (Tablas 1, 2, 3 y 4). En algunas especies, se han incluido registros recientes que pueden resultar de interés.

Palabras clave: Catálogo; Halictini; Halictus; Lasioglossum; Península Ibérica; Islas Canarias; sinonimia; distribución.

\section{ABSTRACT}

C. Ornosa, M. López-Goñi, F. Torres \& D. Romero. 2013. A catalogue of the lberian Peninsula and Canary Islands bees of the Halictini Halictus Latreille, 1804 and Lasioglossum Curtis, 1833 (Hymenoptera, Apoidea, Halictidae). Graellsia, 69(2): 247-274 (in Spanish).

The current work is a catalogue of the Halictini genera Halictus Latreille, 1804 and Lasioglossum Curtis, 1833 in the Iberian Peninsula and the Canary Islands. It includes the taxa, their synonymies, discussion, when necessary, and their geographic distribution as well (Tables 1, 2, 3 y 4). Some recent records of several species have been included too. The results (Table 1) indicate that this fauna is composed of 143 species and 161 subspecies: 126 appear in the Iberian Peninsula and 17 in the Canary Islands, where 6 of them and 17 subspecies are endemic, out of a total of 29 subespecies; 9 of the Canary species are present in both territories.

Key words: Catalogue; Halictini; Halictus; Lasioglossum; Iberian Peninsula; Canary Islands; synonymy; distribution.

* Departamento de Zoología y Antropología Física. Facultad de Biología. Universidad Complutense. c/ José Antonio Nováis, 2. E-28040 Madrid, España. E-mail: paddy@bio.ucm.es

** Departamento de Biología Animal, Ecología, Parasitología y Edafología. Universidad de Salamanca. Campus Miguel de Unamuno s/n. E-37071 Salamanca, España. E-mail: torres@usal.es

1 Subvencionado en parte por el Proyecto CGL2012-34897 


\section{Introducción}

Los Halictini de los géneros Halictus Latreille, 1804 y Lasioglossum Curtis, 1833 son un grupo homogéneo y complejo de abejas, cuya taxonomía y sistemática está aún por abordar para nuestro territorio, ya que contiene un alto número de especies, muy parecidas entre sí, sobre todo entre las hembras.

En la mayor parte del siglo XX, siguiendo las obras más antiguas anteriores (Brullé, 1844; Morawitz, 1879; Vachal, 1895), los componentes del grupo fueron considerados como un único género, lo que dificultó más aún su identificación y organización, si se tiene en cuenta, además, que tales publicaciones normalmente carecían de ilustraciones; tal fue el caso de los pocos especialistas que estudiaron las faunas ibérica y canaria de estos insectos (Brullé, 1844; Vachal, 1895; Pérez, 1895b; Blüthgen, 1923a, 1923b, 1924a, 1924b, 1925, 1937; Dusmet, 1921), si bien debe resaltarse la labor básica realizada por Blüthgen en tales publicaciones, en especial la de 1924 (Blüthgen, 1924b). Trabajos posteriores acometieron este conocimiento $\mathrm{y}$, aunque son numerosos en el continente para faunas europeas o más generales (Ebmer, 1969, 1970, 1971, 1972a, 1972b, 1973, 1975a, 1975b, 1975c, 1976a, 1976b, 1976c, 1978, 1985, 1987, 1988a, 1988b, 1995, 2003, 2011; Pesenko, 2005; Pauly, 2011a-e; Pauly et. al., 2011), son escasos los que abordan los Halictini ibéricos y canarios y normalmente lo hacen desde perspectivas faunísticas (Suárez \& Martínez, 1972; Warncke, 1975a; Ebmer, 1979; Pérez-Íñigo, 1984b; Espeso \& Gayubo, 1988; Diniz, 1989; Gayubo et al., 1990; Hohmann et al., 1993) o como recopilaciones en general (Ortiz-Sánchez, 2006 y 2011).

La distribución geográfica de la familia Halictidae es cosmopolita, con representación en todos los continentes, excepto en la Antártida (Michener, 1974 y 2007). Incluye más de 2.400 especies de las que se estima que unas 830 son eusociales y, junto con la mayor parte de los representantes de Apidae, por tanto, son muy relevantes desde la perspectiva de la evolución de la sociabilidad (Michener, 1974 y 2007; Danforth et al., 2003; Schwarz, et al, 2007), ya que la mayoría de grupos eusociales, como Halictus y Lasioglossum, se encuentran en el Hemisferio Norte (Michener, 2007). La tribu Halictini es la más amplia y diversificada, dentro de los Halictidae en general, y de las abejas (Apoidea) en particular y son varios de sus géneros (Halictus Latreille, 1804, Lasioglossum Curtis, 1833, Augochlora Smith, 1853,
Augochlorella Sandhouse, 1937 y Augochloropsis Cockerell, 1897, por ejemplo) los que incluyen especies eusociales. Además, se dan todos los niveles de sociabilidad conocidos hasta ahora: comportamientos eusociales sencillos, complejos e incluso reversiones (Parker, 1991; Michener 2007; Schwarz, et al., 2007; Wcislo \& Danforth, 2007).

Trabajos recientes sobre Halictini, por otro lado, utilizan taxonomía molecular (Danforth, 1999; Danforth \& Ji, 2001, Danforth et al., 2003, Patiny \& Michez, 2006; Schwarz et al., 2007) o integral, combinando molecular y morfológica (Gibbs, 2009), pero la base de cualquier estudio, sea cual sea la metodología empleada, requiere saber en una primera instancia, qué géneros y especies existen en un territorio determinado para su revisión actualizada y para valorar las posibles agrupaciones, su distribución, la entidad de los taxones que contienen y sus relaciones filogenéticas. En este contexto, se incluye el trabajo ahora presentado, el catálogo base de las especies ibéricas y canarias de Halictini, de los géneros Halictus y Lasioglossum, sobre el que se asentará el conocimiento ulterior de los Halictidae ibéricos. El objetivo fundamental de este estudio es, por lo tanto, conocer y clarificar cuáles son sus componentes en los territorios ibérico y canario y establecer su distribución actual.

\section{Material y métodos}

El presente catálogo incluye los taxones de Halictini de los géneros Halictus y Lasioglossum del ámbito biogeográfico ibérico, es decir, de la Península Ibérica y Baleares y, dadas ciertas precisiones necesarias, se han tratado también las especies de las Islas Canarias.

Se han revisado todos las listas sinonímicas o faunísticas, recopilaciones y catálogos que contienen fauna ibérica y canaria de Halictini, de los que se han extraído las citas incluidas en el texto, tanto de los antiguos (Brullé, 1844; Vachal, 1895; Pérez, 1903: Dusmet, 1921; Blüthgen, 1923a, 1923b, 1923c, 1924a, 1924b, 1937; Ceballos, 1956), como más recientes (Warncke, 1975a; Ebmer, 1979; PérezIñigo, 1984a y 1984b; Espeso \& Gayubo, 1988; Diniz, 1989; Gayubo et al., 1990; Hohmann et al.,1993; Ortiz-Sánchez, 2006 y 2011) o catálogos o textos foráneos sobre faunas próximas (Pesenko, 1984b, 2005 y 2007; Warncke, 1975b, 1981 y 1984; Blank \& Kraus, 1994; Pagliano, 1988; Schwarz et 
al., 1996; Ebmer, 1985, 1999 y 2003; Louadi, 1999; Straka et al., 2007; Dikmen et al., 2011). Se ha utilizado, salvo rara excepción, la bibliografia original, rastreando las publicaciones desde la primera cita de cada taxón hasta las más recientes.

Se ha procedido igualmente para la distribución y categorías corológicas, si bien debe señalarse que, en el texto, se incluyen solo los rangos generales y no se pormenorizan citas concretas más que en casos puntuales. Dada la abundante bibliografía utilizada, no se especifican más referencias que aquellas que la discusión o anotaciones concretas sobre las distintas interpretaciones de ciertos taxones han requerido. Se han incluido, asimismo, nuevos registros recientes de ciertas especies, que pueden resultar de interés.

Dado que no existe un acuerdo general en la concepción genérica y subgenérica del grupo (Parker, 1991; Pesenko 1984a, 1984b, 1984c, 2000, 2004, 2005 y 2007; Straka et al., 2007; Wcislo \& Danforth, 2007; Pauly, 2011a-e, etc.) y que se dan múltiples variaciones al respecto, desde el punto de vista taxonómico, se sigue en general a Michener (2007), para su visión de los géneros y se ha optado por la visión actual más sencilla, que coincide parcialmente, en unos u otros casos, con las distintas agrupaciones establecidas por Pesenko (1984b y 2000) y Ebmer (1969-2011). En corología, se sigue la visión clásica de La Greca (1990), actualizada por Vigna Taglianti et al. (1999), pero con ciertos matices que el ámbito tratado exige en cada caso.

Con el término latino auctt., que aparece en el listado tras el nombre de varias especies, se alude a denominaciones y/o combinaciones empleadas por ciertos autores en un sentido diferente al establecido por el autor original. Igualmente, se emplea el término partim pues, aunque las sinonimias no lo son en parte, la diferencia entre sexos en apoideos ha hecho que la confusión en ocasiones solo haya sido parcial, por lo que, solo a título informativo, se señala.

\section{Resultados y discusión}

Las faunas ibérica y canaria de Halictini de los géneros Halictus y Lasioglossum contienen 143 especies y 161 subespecies: 126 de las especies se conocen en la fauna ibérica y 17 en las Islas Canarias y, de estas, 6 especies y 17 subespecies son endémicas, de un total de 29 subespecies presentes en las islas. De las 17 especies canarias, 9 de ellas están presentes también en el territorio ibérico.

\section{Familia HALICTIDAE Subfamilia HALICTINAE}

Género Halictus Latreille, 1804

Halictus Latreille, 1804. Nouv. Dict. Hist. Nat., 24: 182

ESPECIE TIPO: Apis quadricincta Fabricius, 1776, por designación de Richards, 1935.

Género holártico y tropical, casi cosmopolita.

Subgénero Halictus Latreille, 1804

Halictus Latreille, 1804. Nouv. Dict. Hist. Nat., 24: 182

ESPECIE TIPO: Apis quadricincta Fabricius, 1776, por designación de Richards, 1935

Odontalictus Robertson, 1918. Ent. News., 29: 91

ESPECIE TIPO: Halictus ligatus Say, 1837, por designación original y monotipia

Monilapis Cockerell, 1931. Ann. Mag. Nat. Hist., 7 (10): 529

ESPECIE TIPO: Hylaeus tomentosus Eversmann, $1852=$ Apis flavipes Panzer, 1798 (non Fuesslin, 1775, non Fabricius, 1787 ) = Andrena compresa Walckenaer, 1802, (nomen novum para Apis flavipes Panzer, 1798) = Halictus compresus Walckenaer, 1802, (nomen dubium) $=$ Hylaeus tomentosus Herrich-Schäffer, 1840, (nomen novum para Apis flavipes Panzer, 1798)

Prohalictus Pesenko, 1984. Ent. Obozr., 63: 346

ESPECIE TIPO: Apis rubicunda Christ, 1791, por designación original

Nealictus Pesenko, 1984. Ent. Obozr., 63: 346

ESPECIE TIPO: Halictus parallelus Say, 1837, por designación original

Ramalictus Pesenko, 1984. Ent. Obozr., 63: 347

ESPECIE TIPO: Halictus latisignatus Cameron, 1908, por designación original y monotipia

Platyhalictus Pesenko, 1984. Ent. Obozr., 63: 347

ESPECIE TIPO: Halictus minor Morawitz, 1876, por designación original

Acalcaripes Pesenko, 1984. Ent. Obozr., 63: 347-348

ESPECIE TIPO: Halictus patellatus Morawitz, 1874, por designación original

Tytthalictus Pesenko, 1984. Ent. Obozr., 63: 348

ESPECIE TIPO: Halictus maculatus Smith, 1848, por designación original

Argalictus Pesenko, 1984. Ent. Obozr., 63: 348

ESPECIE TIPO: Hylaeus senilis Eversmann, 1852, por designación original

Hexataenites Pesenko, 1984. Ent. Obozr., 63: 348

ESPECIE TIPO: Apis sexcincta Fabricius, 1775, por designación original

Lampralictus Pesenko, 1984. Ent. Obozr., 63: 348

ESPECIE TIPO: Halictus modernus Morawitz, 1876, por designación original y monotipia

Subgénero con distribución paleártica. 


\section{Halictus (Halictus) asperulus Pérez, 1895}

Halictus asperulus Pérez, 1895. Esp. nouv. Mellif. Barbarie: 65 Halictus rugosulus Pérez, 1895. Esp. nouv. Mellif. Barbarie: 52 (non Schenck, 1853)

Especie paleártica, que llega hasta Asia central.

Halictus (Halictus) brunnescens (Eversmann, 1852)

Hylaeus brunnescens Eversmann, 1852. Bull. Soc. Imp. Nat. Moscou, 25(3): 36

Halictus quadricinctus var. maximus Friese, 1916. Dt. Ent. Z., 1916: 29

Halictus quadricinctus var. aegyptiacus Friese, 1916. Dt. Ent. Z., 1916: 30

Especie paleártica occidental, extendida hasta Asia central.

Halictus (Halictus) cochlearitarsis (Dours, 1872)

Lucasius cochlearitarsis Dours, 1872. Revue Mag. Zool., 23: 352 Halictus anomalipes Lebedev, 1910. Rev. Russ. Ent., 10: 310

Especie paleártica occidental, extendida hasta Asia central. Ascher \& Pickering (2012) incluían una cita rusa más oriental, que no se recoge en Dikmen \& Aytekin (2011), Dikmen et al., (2011) ni Pauly et al. (2011).

Halictus (Halictus) consobrinus (Pérez, 1895)

Halictus consobrinus Pérez, 1895. Esp. nouv. Mellif. Barbarie: 52 Halictus maroccanus Blüthgen, 1934. En: Nadig, Jber. naturf. Ges. Graubünden, 71, (1933): 57

Halictus ifranicola Cockerell, 1937. Am. Mus. Nov., 960: 1

Halictus (Monilapis) rejectus Cockerell, 1937. Am. Mus. Nov., 960: 3

Especie descrita de "Barbarie", pero con distribución mediterránea occidental.

Halictus (Halictus) crenicornis Blüthgen, 1923

Halictus crenicornis Blüthgen, 19232. Konowia, 2 (3-4): 125

Halictus crenicornis Blüthgen, 1924. Mem. R. Soc. Esp. Hist. Nat., 11 (9): 395 syn. nov.

Especie mediterránea noroccidental, descrita de la Península Ibérica; se extiende también por Francia e Italia.

\footnotetext{
Como ya anotó Ebmer (1979), el año de descripción de Halictus crenicornis, y de otra serie de especies presumiblemente establecidas por primera vez por Blüthgen (1924), en realidad no es tal sino 1923. De igual modo que tampoco lo es la referencia correspondiente a 1924, hecho que repetidamente ha inducido a errores posteriores. Puede que la intención de Blüthten sí fuera la de publicar detalladamente dichas especies en su amplia monografía de 1924 (del 25-VIII-1924) [(Mem R. Soc. Esp. Hist. Nat., 11(9)], donde en esta y en las otras especies describe el taxón, indica "nov. sp." e incluye el holotipo, pero en realidad en cada caso se trata de una homonimia que constituye un sinónimo, al haber presentado estas especies también en la revista Konowia antes, en 1923, y señalar el mismo holotipo [(Konowia, 2, fascículos 1-2 y 3-4, datados estos últimos el 1-IX-1923]. Para mayor confusión, en ambas revistas y en todos los casos, aborda cada
}

Halictus (Halictus) compressus (Walckenaer, 1802)

Apis flavipes Panzer, 1798. Faunae Insector. Ger: 56, nº 17 (non Fuesslin, 1775), (non Fabricius, 1787)

Andrena compressa Walckenaer, 1802. Fauna Parisien., 2: 105

Melitta quadricincta Kirby, 1802. Monogr. Apum Angliae, 2: 51 (non Fabricius, 1776)

Halictus eurygnathus Blüthgen, 1931. Dt. Ent. Z., 1930: 210, (nomen novum para Hylaeus quadricinctus, sensu Kirby, 1802 y Halictus tomentosus auctt. (non Eversmann, 1852))

Halictus eurygnathopsis Blüthgen, 1936. Mitt. Zool. Mus. Berlin, 21: 293

Hylaeus senex, sensu Warncke, 1984 (non Förster, 1860)

Halictus veneticus Ebmer, 1969. Naturkdl. Jb. Linz, 1969: 162 (non Mózár, 1967)

Especie paleártica. Para Ebmer (1988a e indirectamente 2011) esta especie constituye una sinonimia de una subespecie oriental, la ssp. transvolgensis Pesenko, 1985, de Halictus (Halictus) eurygnathus Blüthgen, 1931, para él una especie válida. La posición adoptada aquí sigue, por ahora, el criterio de ciertas publicaciones recientes (Pesenko, 2005; Dikmen \& Aytekín, 2011; Pauly et al., 2011; Ascher \& Pickering, 2012).

\section{Halictus (Halictus) fulvipes (Klug, 1817)}

Hylaeus fulvipes Klug, 1817. En: Germar, Reise Dalm., 2: 265 Halictus sexcinctellus Dours, 1872. Revue Mag. Zool., 23: 305 Halictus intumescens Pérez, 1895. Esp. nouv. Mellif. Barbarie: 51 Halictus tinicus Strand, 1921. Arch. Naturgesch., 87A (3): 312 Halictus asunicus Strand, 1921. Arch. Naturgesch., 87A (3): 313

Especie mediterránea occidental, incluidas las Islas Canarias, donde es muy frecuente.

Halictus (Halictus) fumatipennis Blüthgen, 1923

Halictus fumatipennis Blüthgen, 19234. Konowia, 2 (1-2): 75 Halictus fumatipennis Blüthgen, 1924. Mem. R. Soc. Esp. Hist. Nat., 11 (9): 393 syn. nov.

Especie endémica de la Península Ibérica.

Halictus (Halictus) jaramielicus Blüthgen, 1923

Halictus jaramielicus Blüthgen, 19235. Konowia, 2 (3-4): 126 Halictus jaramielicus Blüthgen, 1924. Mem. R. Soc. Esp. Hist. Nat., 11 (9): 398 syn. nov.

Especie endémica de la Península Ibérica. Además de en la localidad típica (España, Valladolid: Jaramiel), se

especie nueva en dos páginas distintas, no consecutivas, lo que ha ido sumando distintas posibilidades a su interpretación por los especialistas. Aquí se ha seguido la norma no escrita de indicar, y solo, la primera página en la que está el nombre de la especie nueva con su descripción.

La inclusión por Pérez-Íñigo (1984) de la sinonimia "Halictus scabiosae Brullé, 1840. His. Nat. Il. Canar., II: 87 (non Rossi, 1790)“ se trata de un error, ya que en dicha publicación Brullé (1844, p. 87, y no 1840) no establece una nueva especie en este caso, sino que se refiere a la especie de Rossi (1790), con la que compara el ejemplar que estudia.

Por otro lado, el año de esta publicación (Brullé, 1844) no es 1839, ni 1840 , como aparece en las distintas publicaciones que transcriben la incluida por Ebmer (1972b), sino 1844, que fue el último año en que aparece en 
conoce por otra cita de la provincia de Segovia (Espeso \& Gayubo, 1988) no incluida por Pauly et al. (2011).

Halictus (Halictus) maculatus Smith, 1848

Halictus interruptus Lepeletier, 1841, Hist. Nat. Insectes Hyménopt., 2: 270 (non Panzer, 1798)

Halictus maculatus Smith, 1848. Zoologist, 6: 2172

Especie paleártica occidental, extendida hasta Asia central.

Halictus (Halictus) patellatus Morawitz, 1874

Halictus patellatus Morawitz, 18746 ${ }^{6}$ Horae Soc. entomol. Ross., 10 (1873-1874): 162

Halictus patellatus, sensu Blüthgen, 1938 (non Morawitz, 1874)

Especie paleártica, con dos subespecies (Pesenko, 2005) de las que la población ibérica pertenece a Halictus patellatus taorminicus Strand, 1921.

Halictus (Halictus) patellatus taorminicus Strand, 1921

Halictus taorminicus Strand, 1921. Arch. Naturgesch., 87A (3): 310

Subespecie paleártica occidental, distribuida por el norte de Africa, Europa (desde Portugal y España, hasta el Volga y Austria oriental), el norte del Cáucaso y Asia suroccidental (hasta el norte de Irán). La citas de la especie (España: Provincias de Barcelona, Gerona, Jaén, Segovia y Zaragoza) que incluían Espeso \& Gayubo (1988) deben considerarse aquí.

\section{Halictus (Halictus) pyrenaeus Pérez, 1903}

Halictus pyrenaeus Pérez, 1903. Proc. verb. Soc. Linn. Bordeaux, 58: 208

Endemismo pirenaico muy raro, descrito sin precisar la localidad del holotipo y conocido solo por otros dos registros: de Francia (Gripp, Hautes Pyrénées) (Ebmer, 1972a) y de España (Pic du Pepolin, Huesca) (Pauly et al., 2011).

el "Tome Deuxième", la"Deuxème partie, Contenant la Zoologie" de esta obra general sobre las Islas Canarias y que correspondió a los años comprendidos entre 1836 y 1844 ("MDCCCXXXVI-XLIV"), como puede observarse en la portada del mismo. El error que se arrastra desde entonces radica en que el tomo de 1840 (el número 3, "Troisiéme" "Contenant la Geographie Botanique") debió salir antes a la luz, como puede deducirse de la"Introduction" del volumen 2, a cargo de uno de los editores (Webb y Berthelot, 1844, p. 3), dando lugar a tal malinterpretación. Brullé solo intervino en el tomo 2, segunda parte.

4,5 En relación con el año y referencia exactos de publicación de Halictus fumatipennis Blüthgen, 1923, véase la nota al pie número 2.

$6 \quad$ Véanse Kerzhner (1984) y Ornosa (2001) en relación con los años de publicación de ciertos volúmenes de la revista Horae Societatis Entomologicae Rossicae.
Halictus (Halictus) quadricinctus (Fabricius, 1776)

Apis quadricincta Fabricius, 1776. Gen. Ins.: 247

Apis hortensis Geoffroy, 1785. En: Fourcroy (Ed.), Entomol.

Paris., 2: 446

Halictus quadristrigatus Latreille, 1805. Hist. Nat. Crust. Insect., 13: 364

Hylaeus grandis Illiger, 1806. Mag. Insektenkd. (Illiger), 5: 57

Halictus ecaphosus Walckenaer, 1817. Mém. Hist. Nat. Abeill. Solit.: 58

Especie paleártica.

Halictus (Halictus) quadripartitus Blüthgen, 1923

Halictus quadripartitus Blüthgen, 19237. Konowia, 2 (3-4): 128

Halictus quadripartitus Blüthgen, 1924. Mem. R. Soc. Esp.

Hist. Nat., 11 (9): 397 syn. nov.

Especie endémica de la Península Ibérica.

Halictus (Halictus) rubicundus (Christ, 1791)

Apis rubicunda Christ, 1791. Naturg Ins.: 109

Halictus nidulans Walckenaer, 1817. Mém. Hist. Nat. Abeill. Solit: 69

Halictus lerouxi Lepeletier, 1841. Hist. Nat. Insectes Hyménopt., 2: 272

Hylaeus tomentosus Eversmann, 1852. Bull. Soc. Imp. Nat. Moscou, 25: 37

Halictus quadrifasciatis Smith, 1870. Entomologist's Ann.: 25 Halictus lerouxi ruborum Cockerell, 1898. Canad. Entomol., 30: 52

Halictus nesiotis Perkins, 1922. Entomol. Mon. Mag., 58: 273 (non Crawford, 1918)

Halictus rubicundus laticinctus Blüthgen, 1923. Konowia, 2 (1-2): 70

Halictus lupinelli Cockerell, 1936. Pan. Pac. Ent., 12: 158

Halictus rubicundus mongolensis Blüthgen, 1936. Mitt. Zool. Mus. Berlin, 21: 302

Halictus frater Pesenko, 1984. En: Korotyaev, Nasekom. Mongol., 9: 469

Especie holártica. En el sur de Europa suele presentar tendencia orófila.

Halictus (Halictus) scabiosae (Rossi, 1790)

Apis scabiosae Rossi, 1790. Faun. Etrusc., 2: 105

Hylaeus alternans Fabricius, 1793. Syst. Entomol., 2: 203

Halictus zebrus Walckenaer, 1817. Mém. Hist. Nat. Abeill.

Solit: 68

Halictus griseozonatus Dours, 1872. Revue Mag. Zool., 23: 308

Halictus scabiosae powelli Cockerell, 1931. Ann. Mag. Nat.

Hist., 7 (10): 531

Especie paleártica, muy frecuente en la Península Ibérica y Baleares.

En relación con el año y referencia exactos de publicación de Halictus fumatipennis Blüthgen, 1923, véase la nota al pie número 2. 
Halictus (Halictus) senilis (Eversmann, 1852)

Hylaeus senilis Eversmann, 1852. Bull. Soc. Imp. Nat. Moscou, 25: 38

Halictus fucosus Morawitz, 1876. En: Fedtschenko, Izv. Imp. Obsetsva. Lûbit. Estestv. Antropol. Etnogr., 21 (2): 230

Halictus albarius Pérez, 1895. Esp. nouv. Mellif. Barbarie: 51 Halictus bivinctus Vachal, 1902. Rev. Russ. Ent., 2: 226

Halictus aegypticola Strand, 1909. Arch. Naturgesch., 75, I (1): 21

Halictus libanensis Pérez, 1912. Bull. Soc. Amis Sci. Nat. Rouen, 47 (1911): 84

Especie paleártica. En el territorio ibérico se conoce solo y escasamente del sur peninsular (Almería) y, en Canarias, en las islas de Lanzarote y la Graciosa.

Halictus (Halictus) sexcinctus (Fabricius, 1775)

Apis sexcincta Fabricius, 1775. Syst. Entomol.: 387 (non Kirby, 1802)

Apis ichneumonea Christ, 1791. Naturg. Ins.: 198 (non Linnaeus, 1758)

Hylaeus sexcinctus Fabricius, 1793. Syst. Entomol., 2: 304

Hylaeus arbustorum Panzer, 1797. Faunae Insector. Ger., 4: 14,46

Andrena rufipes Spinola, 1806. Insector. Liguriae, 1: 123

Especia paleártica occidental, extendida hasta Asia central. En el territorio ibérico se conoce en la mitad norte peninsular y en Baleares.

Halictus (Halictus) simplex Blüthgen, 1923

Halictus marchali Vachal, 1891. Rev. Ent., 10: 65 (nomen dubium)

Halictus simplex Blüthgen, 1923. Konowia, 2 (3-4): 127

Halictus ibex Warncke, 1973. Bull. Soc. R. Sci. Liége, 42: 282, (nomen novum para Halictus simplex Blüthgen, 1923) (non Robertson, 1901)

Especie paleártica occidental, que llega hasta Irán.

Halictus (Halictus) tetrazonius (Klug, 1817)

Hylaeus tetrazonius Klug, 1817. En: Germar, Reise Dalm., 2: 265

Halictus furcatus Blüthgen, 1925. Arch. Naturgesch., 90A (10): 94

Halictus galilaeus Blüthgen, 1955. Bull. Res. Counc. Isr., 5 (B): 15

Halictus pannonicus Ebmer, 1969. Naturkdl. Jb. Linz, 1969: 165 (non Osmia pannonica Zilahi Kiss, 1915)

Especie paleártica. Según Ortiz-Sánchez (2006) -como Halictus (Halictus) aff. tetrazonius (Klug, 1817)- en España aparece en Andalucía; además existe en el cuadrante nororiental y en el centro peninsular.
Halictus (Halictus) tridivisus Blüthgen, 1923

Halictus tridivisus Blüthgen, 19238. Konowia, 2 (3-4): 129

Halictus tridivisus Blüthgen, 1924. Mem. R. Soc. Esp. Hist. Nat., 11 (9): 396 syn. nov.

Especie endémica de la Península Ibérica, conocida en la mitad norte de España peninsular. La abreviatura "H" (provincia de Huelva, extremo sur ibérico) que recogen Espeso \& Gayubo (1988) se trata de una errata de transcripción de la provincia de Huesca $(\mathrm{Hu})$, de una de las localidades típicas ("Panticosa") de las que proceden dos de los paratipos (Blüthgen, 1923 y 1924b).

Subgénero Seladonia Robertson, 1918

Seladonia Robertson, 1918. Ent. News, 29: 91

ESPECIE TIPO: Apis seladonia Fabricius, 1794, por designación original.

Pachyceble Moure, 1940. Arch. Zool. Est. S. Paulo, 2: 54

ESPECIE TIPO: Pachyceble lanei Moure, 1940, por designación original.

Subgénero repartido por las regiones Paleártica, Etiópica y Oriental (Michener, 2007).

Halictus (Seladonia) confusus Smith, 1853

Halictus confusus Smith, 1853. Cat. Brit. Hym. Brit. Mus., 1: 70 Halictus alpinus Alfken, 1907. Z. Syst. Hymenopt. Dipterol., 7: 205

Especie holártica, muy politípica. En la Península Ibérica tiene principalmente carácter orófilo y aparece diferenciada en dos subespecies que se detallan a continuación.

Halictus (Seladonia) confusus perkinsi Blüthgen, 1926 Halictus perkinsi Blüthgen, 1926. Dt. Ent. Z. (1925): 417

Subespecie distribuida por Europa, desde los Pirineos, en España, hasta los Urales. En el territorio ibérico es la más abundante, repartida por la mitad norte peninsular.

La abreviatura " $\mathrm{H}$ " (que normalmente se le asigna a la provincia de Huelva, situada en el extremo sur ibérico), incluida en la distribución de la especie que recopilan Espeso \& Gayubo (1988) es un error de transcripción de la provincia de Huesca $(\mathrm{Hu})$, donde sí se conocía previamente Halictus confusus (Blüthgen, 1924b y 1925; Ebmer, 1979 y 1988b).

Halictus (Seladonia) confusus glacialis Ebmer, 1979

Halictus confusus glacialis Ebmer, 1979. Linzer biol. Beitr., 11 (1): 121

Subespecie de altitud, endémica de la Sierra de Guadarrama (Madrid), España.

En relación con el año y referencia exactos de publicación de Halictus fumatipennis Blüthgen, 1923, véase la nota al pie número 2. 
Halictus (Seladonia) gavarnicus Pérez, 1903

Halictus gavarnicus Pérez, 1903. Proc. verb. Soc. Linn. Bordeaux, 58: 210

Especie euroasiática con tendencia orófila en el sur (Pirineos, Alpes, Cáucaso, etc.) y presentando distintas subespecies en los diferentes sistemas montañosos. En el entorno ibérico solo se conoce en los Pirineos en su forma nominal.

Halictus (Seladonia) gemmeus Dours, 1872

Halictus gemmeus Dours, 1872. Revue Mag. Zool., 23: 310

Especie mediterránea.

Halictus (Seladonia) kessleri Bramson, 1879

Halictus kessleri Bramson, 1879. Bull. Soc. Imp. Nat. Moscou, 54: 286

Osmia pannonica Zilahi-Kiss, 1915. Rovartani Lapok, 22: 83-84

Halictus kessleri Blüthgen, 1921. Dt. Ent. Z., 1920: 291

Halictus kessleri nebulosus Warncke, 1975. Polski Pismo Entomol., 45: 119

Especie normediterránea. En el área estudiada, Ebmer (1988a) mencionaba una población aislada en los Pirineos.

Halictus (Seladonia) leucaheneus Ebmer, 1972

Halictus leucaheneus Ebmer, 1972. Mitt. Zool. Mus. Berlin, 48: 225

Especie paleártica. De las tres o cuatro subespecies que se reconocen, en el territorio estudiado aparece la siguiente:

Halictus (Seladonia) leucaheneus arenosus Ebmer, 1976 Halictus fasciatus auctt. (non Nylander, 1848)

Halictus leucaheneus arenosus Ebmer, 1976. NachrBl. bayern. Ent., 25: 2, (nomen novum para Halictus fasciatus auctt. (non Nylander, 1848))

Subespecie europea, extendida hasta los Pirineos en el sur y hasta Macedonia, por el este.

Halictus (Seladonia) nivalis Ebmer, 1985

Halictus nivalis Ebmer, 1985. Linzer biol. Beitr., 17 (1): 201

Especie descrita de Sierra Nevada (España) y endémica de la Península Ibérica (Ebmer, 2011). Ascher \& Pickering (2012) incluían una cita de Linz (Austria) y Pauly (2011a) le concedía rango de subespecie de Halictus leucaheneus Ebmer, 1972.

Halictus (Seladonia) seladonius (Fabricius, 1794)

Apis seladonia Fabricius, 1794. Syst. Entomol., 4: 460

Halictus geminatus Pérez, 1903. Proc. verb. Soc. Linn. Bordeaux, 58: 209
Especie paleártica, frecuente en la Península Ibérica.

Halictus (Seladonia) smaragdulus Vachal, 1895

Halictus smaragdulus Vachal, 1895. An. Soc. Esp. Hist. Nat., 24: 150

Halictus subauratus Lepeletier, 1825. Encycl. Method. Insect., 10: 797

Halictus barcelonicus Pérez, 1903. Proc. verb. Soc. Linn. Bordeaux, 58: 211

Halictus subauratus forma vinulus Blüthgen, 1923. Arch. Naturgesch., 89A (5): 300

Halictus morinellus Warncke, 1975. Polski Pismo Entomol., 45: 118

Halictus buteus Warncke, 1975. Polski Pismo Entomol., 45: 119

Especie paleártica occidental, descrita de Sevilla (España), que llega hasta las montañas de Asia central. Ascher \& Pickering (2012) también la incluían en Australia Oriental.

NuEvos REgISTROS: España: Segovia: Sierra de Guadarrama, Venta de los Mosquitos, 1400 m, 14-VII-2009, 1 ㅇ ; 24-IX2009; Ornosa et al., leg.; UCM. Sobre Jacobaea vulgaris Gaertn. (1754). Pérez-Íñigo (1984b) la citó de otras localidades de esta misma sierra.

Halictus (Seladonia) subauratus (Rossi, 1792)

Apis subaurata Rossi, 1792. Mant. Ins., 1: 144

Halictus virescens Lepeletier, 1841. Hist. Nat. Insectes Hyménopt., 2: 279

Halictus gramineus Smith, 1849. Zoologist, 7: 58

Halictus meridionalis Morawitz, 1874. Horae Soc. entomol. Ross., 10 (1873-1874): 170

Halictus subauratus syrius Blüthgen, 1933. Dt. Ent. Z.: 72

Especie paleártica occidental.

Halictus (Seladonia) tumulorum (Linnaeus, 1758)

Apis tumulorum Linnaeus, 1758. Syst. Nat. (Ed.10), 1: 574 (non Panzer, 1804)

Apis flavipes Fabricius, 1787. Mant. Ins., 1: 305 (non Fuesslin, 1775)

Halictus fasciatus Nylander, 1848. Not. Sällsk. Fauna Flora Fenn. Förh., (Adnot.) 1: 275

Halictus tumulorum devitidatus Strand, 1910. Nyt. Mag. Naturvid., 48: 336

Halictus ferripennis Cockerell, 1929. Ann. Mag. Nat. Hist., 4 (10): 586

Especie paleártica. En la Península Ibérica se reparte, con carácter orófilo, por el norte, con alguna penetración en el Sistema Central. Para Warncke (1981), Halictus fasciatus Nylander, 1848, constituía una especie válida.

Subgénero Vestitohalictus Blüthgen, 1961

Vestitohalictus Blüthgen, 1961. Beitr. Nat. For. Südw., 19: 287 EsPECIE TIPO: Halictus vestitus Lepeletier, 1841. Hist. Nat. Insectes Hyménopt., 2: 281, por designación original. 
Halictus (Vestitohalictus) balearicus Pérez, 1903

Halictus balearicus Pérez, 1903. Proc. verb. Soc. Linn. Bordeaux, 58: 209

Especie endémica de Baleares. Pauly (2011c) y Ascher \& Pickering (2012), a diferencia de Pérez-Íñigo (1984a), Ebmer (1988a) y Ortiz-Sánchez (2011) le dan tratamiento subespecífico de Halictus (Vestitohalictus) pollinosus.

Halictus (Vestitohalictus) concinnus (Brullé, 1844) ${ }^{9}$

Halictus concinnus Brullé, 1844. Hist. nat. Il. Canar., 2 (Partie 2) (1836-1844): 88

Especie endémica de Canarias. Muy frecuente en las distintas islas del archipiélago.

Halictus (Vestitohalictus) microcardia Pérez, 1895

Halictus microcardia Pérez, 1895. Ann. Soc. Entomol. France, 43: 193

Especie endémica de Baleares. La cita original se hizo de "Fuerteventura" (Canarias), además de "Baleares", y más tarde se recolectó en Ibiza (Ebmer, 1975c y 2011). Durante un tiempo (Ebmer, 1972 y 1988a; Pérez-Iñnigo, 1984a) fue considerada como sinonimia de Halictus (Vestitohalictus) concinnus, especie exclusiva de las Islas Canarias.

Halictus (Vestitohalictus) pollinosus Sichel, 1860

Halictus pollinosus Sichel, 1860. Ann. Soc. Entomol. France, 8 (3): 763

Especie paleártica. En la fauna ibérica, de entre las cuatro o cinco subespecies que se diferencian (Ebmer, 1988a; Pauly, 2011c; Ascher \& Pickering, 2012), se encuentra Halictus pollinosus cariniventris Morawitz, 1876.

\section{Halictus (Vestitohalictus) pollinosus cariniventris Mora-} witz, 1876

Halictus cariniventris Morawitz, 1876. En: Fedtschenko, Izv. Imp. Obsetsva. Lûbit. Estestv. Antropol. Etnogr., 21 (2): 226

Halictus cariniventris var. creticola Strand, 1921. Arch. Naturgesch., 87A (3): 314

Halictus cariniventris flavotectus Cockerell, 1922. Ann. Mag.

Nat. Hist., 10 (9): 550

Halictus pollinosus limissicus Blüthgen, 1937. Konowia, 16: 43

Subespecie que abarca el territorio europeo continental y Asia central hasta Mongolia (Ebmer, 1988b). Pauly (2011c) consideraba a Halictus cariniventris creticola $y$ Halictus pollinosus limissicus como dos subespecies válidas.

\footnotetext{
Véase la segunda parte de la nota 3, en relación con el año de publicación de Brullé (1844).
}

Halictus (Vestitohalictus) vestitus Lepeletier, 1841

Halictus vestitus Lepeletier, 1841. Hist. Nat. Insectes Hyménopt., 2: 281

Halictus pulvereus Morawitz, 1874. Horae Soc. entomol. Ross., 10 (1873-1874): 168

Halictus velatus Pérez, 1895. Esp. nouv. Mellif. Barbarie: 53

Especie euroasiática principalmente normediterránea en su forma nominal, aunque, como Halictus velatus, fue citada de "Barbarie" (Pérez, 1895a). Como subespecie, Halictus vestitus velatus, contiene, además, a la población que aparece en las Baleares. Una tercera subespecie, ssp. tectus Radoszkovski 1875, se reparte por el resto de Eurasia hasta Mongolia.

Género Lasioglossum Curtis, 1833

Lasioglossum Curtis, 1833. Brit. Ent. 10: 448

ESPECIE TIPO: Melitha xanthopus Kirby, 1802

Género holártico y tropical, casi cosmopolita.

Subgénero Lasioglossum Curtis, 1833

ESPECIE TIPO: Melitha xanthopus Kirby, 1802

Subgénero holártico y mesoamericano.

Lasioglossum (Lasioglossum) aegyptiellum (Strand, 1909) Halictus morbillosus forma aegyptiellus Strand, 1909. Arch. Naturgesch., 75, I (1): 11

Halictus divergens Pérez, 1911. Bull. Soc. Amis Sci. Nat. Rouen, 46 (1910): 46

Halictus orontis Cockerell, 1937. Af. Bees Genera Cer. Hal. Meg.: 97

Halictus platycestus auctt. (non Dours, 1872)

Especie descrita de Egipto, con distribución mediterránea, extendida hasta Turkmenistán e Irán. Su presencia en la Península Ibérica fue recogida por Pérez-Íñigo (1984a) y descartada por Ebmer (1988a). No obstante existe una cita de ese mismo año del sur de España (Herrera, 1988), que se refleja en Polaszek (2004) y Ascher \& Pickering (2012) y no en Pauly (2011e). Su existencia en la Península Ibérica debería confirmarse con nuevas capturas.

Lasioglossum (Lasioglossum) albocinctum (Lucas, 1849) Halictus albocinctus Lucas, 1849. Explor. Sci. Algérie (Artic.), 3: 183

Halictus platycestus Dours, 1872. Revue Mag. Zool., 23: 306

Halictus separandus Frey-Gessner, 1903. Faun. Insect. Helvet., 1: 202

Halictus phanerodontus Cockerell, 1931. Ann. Mag. Nat. Hist., 7 (10): 532

Halictus bimaculatus auctt.

Lasioglossum albomaculatum auctt.

Especie mediterránea occidental, incluidos Grecia y el sur de Suiza y de Alemania (Ebmer, 1988a). 
Tal como señalaba Ebmer (1988a), la denominación de Halictus bimaculatus Lucas, 1846, se trataba de un error de identificación, de modo que su inclusión por Pérez-Íñigo (1984a) en el catálogo de halíctidos españoles debe ser rechazada.

\section{Lasioglossum (Lasioglossum) bimaculatum (Dours,} 1872)

Halictus bimaculatus Dours, 1872. Revue Mag. Zool., 23: 349 Halictus perezi Alfken, 1907. Z. Syst. Hymenopt. Dipterol., 3: 203

Especie mediterránea occidental, con tendencia heliófila. La posible existencia en la Península Ibérica de otra subespecie, la ssp. hispanicum Blüthgen, 1931, además de la forma nominal, debe ser confirmada (Ebmer, 1979; Pauly, 2011d).

Lasioglossum (Lasioglossum) breviventre (Schenck, 1853)

Halictus breviventris Schenck, 1853. Jahrb. Ver. Natur. Nassau, 9: 151

Halictus quadrifasciatus Schenck, 1875 Dt. Ent. Z., 19: 321 (non Smith, 1870)

Halictus chalconotus Pérez, 1895. Esp. nouv. Mellif. Barbarie: 53

Halictus micans Strand, 1909. Arch. Naturgesch., 75, I (1): 10

Especie europea meridional occidental, rara en la Península Ibérica, donde solo se conoce por escasas citas en la Sierra de Guadarrama y los Pirineos.

Lasioglossum (Lasioglossum) callizonium (Pérez, 1895) Halictus callizonius Pérez, 1895. Esp. nouv. Mellif. Barbarie: 54 Halictus mogadoricus Cockerell, 1937. Am. Mus. Nov., 960: 9

Especie mediterránea.

Lasioglossum (Lasioglossum) chalcodes (Brullé, 1844) ${ }^{10}$ Halictus chalcodes Brullé, 1844. Hist. nat. Il. Canar., 2 (Partie 2) (1836-1844): 87

Endemismo canario (España), representado, además de por la forma nominal, por las subespecies: Lasioglossum (Lasioglossum) chalcodes calderae Ebmer, 1993 y Lasioglossum (Lasioglossum) chalcodes canariense Ebmer, 1993 y Lasioglossum (Lasioglossum) chalcodes gomera (Warncke, 1975).

\section{Lasioglossum (Lasioglossum) chalcodes calderae Ebmer, 1993}

Halictus chalcodes calderae Ebmer, 1993. Veröff. ÜberseeMuseum Bremen, 12: 771

Subespecie endémica de la isla de La Palma.

\footnotetext{
Véase la segunda parte de la nota 3, en relación con el año de publicación de Brullé (1844).
}

Lasioglossum (Lasioglossum) chalcodes canariense Ebmer, 1993

Halictus chalcodes canariense Ebmer, 1993. Veröff. ÜberseeMuseum Bremen, 12: 772

Subespecie endémica de la isla de Gran Canaria.

\section{Lasioglossum (Lasioglossum) chalcodes chalcodes} (Brullé, 1844)

Halictus chalcodes Brullé, 1844. Hist. nat. Il. Canar., 2 (Partie 2) (1836-1844): 87

Sphecodes semiaeneus Brullé, 1844. Hist. nat. Il. Canar., 2 (Partie 2) (1836-1844): 88

Subespecie endémica de las islas de El Hierro y Tenerife.

\section{Lasioglossum (Lasioglossum) chalcodes gomera} (Warncke, 1975)

Halictus chalcodes gomera Warncke, 1975. Vieraea, 4 (1-2) (1974): 206

Subespecie endémica de la isla de La Gomera.

Lasioglossum (Lasioglossum) costulatum (Kriechbaumer, 1873)

Andrena campestris Eversmann, 1852. Bull. Soc. Imp. Nat. Moscou, 25: 20 (no disponible)

Halictus costulatum Kriechbaumer, 1873. Verh. Zool. -Bot. Ges. Wien., 23: 59

Halictus alpestris Morawitz, 1877 ${ }^{11}$. Horae Soc. entomol. Ross., 14: 90

Especie paleártica occidental, hasta Asia central, muy frecuente en la Península Ibérica.

\section{Lasioglossum (Lasioglossum) cristula Pérez, 1895}

Halictus cristula Pérez, 1895. Esp. nouv. Mellif. Barbarie: 54 Halictus mesoleus Cockerell, 1938. Am. Mus. Nov., 997

Halictus cristula ssp. donatus Warncke, 1975. Bull. Ent. Pologne, Wroclaw 45: 93

Especie paleártica occidental, extendida hasta Irán.

Lasioglossum (Lasioglossum) discum (Smith, 1853)

Halictus discus Smith, 1853. Cat. Brit. Hym. Brit. Mus., 1: 70 Halictus morbillosus Kriechbaumer, 1873. Verh. Zool. -Bot. Ges. Wien., 23: 61

Halictus morbillosus glasuvoni Cockerell, 1924. Ann. Mag. Nat. Hist., 14(9): 582

\footnotetext{
En relación con el año de publicación del volumen XIV de Horae Societatis Entomologicae Rossicae, Kerzhner (1984) señalaba 1877 para las páginas de este trabajo de Morawitz, aunque en la portada del volumen aparece su pertenencia a 1878, pero publicado en 1879 (Ornosa, 2001). A pesar de la discrepancia, se mantiene aquí 1877 , para la entrega de las páginas del trabajo de Morawitz, pero haciendo constar en las referencias los tres años que aparecen en el volumen 14, para evitar confusiones.
} 
Especie paleártica occidental, repartida por el norte del Mediterráneo, hasta Israel y por Asia Menor hasta Afganistán.

En la Península Ibérica aparece la siguiente subespecie:

\section{Lasioglossum (Lasioglossum) discum fertoni (Vachal} 1895)

Halictus fertoni Vachal 1895. An. Soc. Esp. Hist. Nat., 24: 149 Lasioglossum pseudomorbillosum Ebmer, 1970. Naturkdl. Jb. Linz, 1970: 30

Subespecie mediterránea occidental.

Lasioglossum (Lasioglossum) laevigatum (Kirby, 1802) Melitta laevigata Kirby, 1802. Monogr. Apum Angliae, 2: 75 Melitta lugubris Kirby, 1802. Monogr. Apum Angliae, 2: 81 Hylaeus semicinctus Förster, 1860. Verh. Naturh. Ver. Preuss. Rheinl. NF, 17: 140

Especie paleártica occidental, distribuida hasta Irán.

\section{Lasioglossum (Lasioglossum) laterale (Brullé, 1832)}

Halictus lateralis Brullé, 1832. Expéd. Scient. Morée, 3(1): 349 Halictus bifasciatus Brullé, 1832. Expéd. Scient. Morée, 3(1): 349 Halictus ticinensis Frey-Gessner, 1903. Faun. Insect. Helvet., 1: 200

Especie mediterránea.

Lasioglossum (Lasioglossum) lativentre (Schenck, 1853)

Hylaeus lativentris Schenck, 1853. Jahrb. Ver. Natur. Nassau, 9: 151

Hylaeus bisbistrigatus Schenck, 1853. Jahrb. Ver. Natur. Nassau, 9: 162

Halictus bicinctus Schenck, 1874. Berl. Ent. Z., 18: 161 (non Apis bicincta Schranck, 1781)

Halictus decipiens Perkins, 1913. Entomol. Mon. Mag., 49: 62

Halictus lativentris sotschica Blüthgen, 1931. Mitt. Zool. Mus. Berl., 17: 350

Especie paleártica occidental, extendida hasta Irán.

Lasioglossum (Lasioglossum) leucozonium (Schrank, 1781)

Apis leucozonia Schrank, 1781. Enum. Insect. Austr.: 406

Especie holártica politípica. En la Península Ibérica existen tres subespecies de las seis que comprende.

\section{Lasioglossum (Lasioglossum) leucozonium cedri Ebmer,} 1976

Lasioglossum leucozonium cedri Ebmer, 1976. Linzer biol. Beitr., 8 (1): 235

Subespecie mediterránea occidental y de Creta y Chipre, que en el territorio ibérico se dispersa por el sur de España (Ebmer, 1988a).

\section{Lasioglossum (Lasioglossum) leucozonium elysium Ebmer, 1979}

Lasioglossum zonulum elysium Ebmer, 1979. Linzer biol. Beitr., 11 (1): 131

Subespecie endémica de la Península Ibérica (Granada, Sierra Nevada).

\section{Lasioglossum (Lasioglossum) leucozonium leucozonium} (Schrank, 1781)

Apis leucostoma Schrank, 1781. Enum. Insect. Austr: 406 Halictus similis Smith, 1853. Cat. Brit. Hym. Brit. Mus., 1: 69 Halictus bifasciatellus Schenck, 1875. Dt. Ent. Z., 19: 322 Halictus leucozonius nigrotibialis Dalla Torre, 1877. Z. Ferdinandeums Tirol Vorarl., 21 (3): 178

Halictus deiphobus Bingham, 1908. Rec. Ind. Mus., 2: 361

Subespecie europea y neártica. En el área ibérica se reparte por el norte del territorio, en los Pirineos, la provincia de Zaragoza y la Sierra de Guadarrama (Ebmer, 1988a).

Nuevos Registros: España: Segovia: Sierra de Guadarrama: Venta de los Mosquitos, 1400 m, 24-VII-2009, 2 우 우, Ornosa et al. Leg.; UCM. Sobre Jacobaea vulgaris Gaertn. (1754). Pérez-Íñigo (1984b) la citó de otras localidades de esta misma sierra.

Lasioglossum (Lasioglossum) majus (Nylander, 1852)

Halictus major Nylander, 1852. Not. Sällsk. Fauna Flora Fenn. Förh., (Adnot.) 2: 240

Halictus lichtensteini Pérez, 1903. Proc. verb. Soc. Linn. Bordeaux, 58: 213

Especie paleártica occidental.

Lasioglossum (Lasioglossum) pallens (Brullé, 1832)

Halictus pallens Brullé, 1832. Expéd. Scient. Morée., 3/1: 350

Halictus lineolatus Lepeletier, 1841. Hist. Nat. Insectes Hyménopt., 2: 275 (non Schrank, 1802)

Halictus cirrhozonius Vachal, 1895. An. Soc. Esp. Hist. Nat., 24: 147

Especie paleártica occidental, extendida hasta Irán.

Lasioglossum (Lasioglossum) perclavipes (Blüthgen, 1934)

Leucasellus perclavipes Blüthgen, 1934. Bol. Soc. entomol. Ital.: 188

Especie mediterránea. De acuerdo con Ebmer (1979), pertenecen a esta especie las citas españolas detalladas por Blüthgen (1924) de Halictus clavipes (Dours, 1872), especie no ibérica. Por lo tanto, la inclusión por Pérez-Íñigo (1984a) de Lasioglossum clavipes (Dours, 1872) en el catálogo de Halíctidos españoles, debe rechazarse. 
Lasioglossum (Lasioglossum) prasinum (Smith, 1848)

Halictus prasinus Smith, 1848. Zoologist, 6: 2169

Hylaeus tomentosus Schenck, 1853. Jahrb. Ver. Naturk. Nassau, 9: 265 (non Herrieh-Schfäfer, 1840)

Hylaeus haemorrhoidalis Schenck, 1853. Jahrb. Ver. Naturk. Nassau, 9: 159

Hylaeus albidus Schenck, 1861. Jahrb. Ver. Naturk. Nassau, 14 (1859): 283, (nomen novum para Hylaeus tomentosus Schenck, 1853 (non Lepetelier, 184))

Halictus semipubescens Dours, 1872. Revue Mag. Zool., 23: 309

Halictus albidulus Schenck, 1874. Berl. Ent. Z., 18: 162, (nomen novum para Hylaeus albidus Schenck, 1861)

Halictus canescens Schenck, 1874. Berl. Ent. Z., 18: 162

Halictus schencki Morawitz, $1877^{12}$. Horae Soc. entomol. Ross., 14: 91 (nomen novum para Hylaeus albidus Schenck, 1861)

Especie paleártica occidental.

Lasioglossum (Lasioglossum) quadrinotatum (Kirby, 1802)

Melitta quadrinotata Kirby, 1802. Monogr. Apum Angliae, 2: 79

Especie paleártica occidental, repartida por la mitad norte del territorio ibérico.

\section{Lasioglossum (Lasioglossum) sexmaculatum (Schenck,} 1853)

Hylaeus sexmaculatus Schenck, 1853. Jahrb. Ver. Naturk. Nassau, 9: 142

Especie paleártica, orófila, rara en la Península Ibérica, solo conocida por un par de citas de Asturias y de la Sierra de Guadarrama (Blüthgen, 1924; Ebmer, 1970 y 1979; PérezÍnigo, 1984b). Pauly (com. per.) considera dudosa la presencia de esta especie en España.

Lasioglossum (Lasioglossum) sexnotatum (Kirby, 1802) Andrena nitida Panzer, 1798. Faunae Insector. Ger., 5: 56, 2 Melitta sexnotata Kirby, 1802. Monogr. Apum Angliae, 2: 82

Especie paleártica.

Lasioglossum (Lasioglossum) subfasciatum (Imhoff, 1832)

Hylaeus subfasciatus Imhoff, 1832. Isis (Oken) Jena: 1199

Halictus rufocinctus Nylander, 1852. Not. Sällsk. Fauna Flora Fenn. Förh., (Adnot.) 2: 239

Hylaeus bifasciatus Schenck, 1853. Jahrb. Ver. Naturk. Nassau, 9: 160 (non Brullé, 1832)

Especie euroasiática, distribuida por Europa media y meridional, extendida hasta Irán, poco frecuente en el territorio ibérico y solo en localidades aisladas de la mitad norte.

\footnotetext{
Véase nota 11
}

Lasioglossum (Lasioglossum) xanthopus (Kirby, 1802)

Apis emarginata Christ, 1791. Nat. gesch. Classif. Nomencl. Insekten: 183, (nomen oblitum)

Melitta xanthopus Kirby, 1802. Monogr. Apum Angliae, 2: 78

Hylaeus derasus Imhoff, 1832. Isis (Oken) Jena,: 1199

Lasioglossum tricingulum Curtis, 1833. Brit. Ent., 10: 448

Hylaeus fulvicrus Eversmann, 1852. Bull. Soc. Imp. Nat. Moscou, 25: 39

Halictus soreli Dours, 1872. Revue Mag. Zool., 23: 302

Especie paleártica occidental.

Lasioglossum (Lasioglossum) zonulum (Smith, 1848)

Halictus zonulus Smith, 1848. Zoologist, 6: 2171

Hylaeus trifasciatus Schenck, 1853. Jahrb. Ver. Naturk. Nassau, 9: 168

Halictus rhenanus Verhoff, 1890. Ent. Nachr., 16: 325

Halictus recepticius Vachal, 1902. Ent. Obozr., 2: 227

Halictus craterus Lovell, 1908. Psyche. Camb., 15: 35

Especie holártica. La subespecie nominal, que es la que se encuentra en el área ibérica, tiene distribución europea.

Subgénero Evylaeus Robertson, 1902

Evylaeus Robertson, 1902. Canad. Entomol., 34: 244 ESPECIE TIPO: Halictus arcuatus Robertson, 1893

Chloralictus Robertson, 1902. Canad. Entomol., 34: 48 ESPECIE TIPO: Halictus cressoni Robertson, 1890

Dialictus Robertson, 1902. Canad. Entomol., 34: 48 ESPECIE TIPO: Halictus anomalus Robertson, 1892

Subgénero holártico y tropical, casi cosmopolita.

Lasioglossum (Evylaeus) aeratum (Kirby, 1802)

Melitta aerata Kirby, 1802. Monogr. Apum Angliae, 2: 58

Halictus semiaeneus Brullé, 1832. Expéd. Scient. Morée, 3(2): 352

Halictus viridiaeneum Blüthgen, 1918. Dt. Ent. Z., 1918: 272

Especie paleártica. Pauly (2013) le asignaba distribución eurosiberiana, aunque Ebmer (1979) mencionaba su presencia en el norte de África y Blüthgen (1924) y Pérez-Íñigo (1984b) la citaban en el norte y el centro peninsular ibérico.

Lasioglossum (Evylaeus) albipes (Fabricius, 1781)

Apis albipes Fabricius, 1781. Spec. Insect., 1: 486

Hylaeus abdominalis Panzer, 1798. Faunae Insector. Ger., 53: 19

Halictus malachurellus Strand, 1909. Arch. Naturgesch., 75, I (1): 40

Halictus albipes var. alpicola Blüthgen, 1920. Dt. Ent. Z., 1920: 284

Especie paleártica. Frecuente en el territorio ibérico, con cierta tendencia orófila. 
Lasioglossum (Evylaeus) albovirens (Pérez, 1895)

Halictus albovirens Pérez, 1895. Esp. nouv. Mellif. Barbarie: 52

Especie paleártica suroccidental, distribuida por el norte de África, las Islas Canarias y el territorio ibérico. Es variable y politípica, por lo que aquí se sigue a Ebmer (1976a y 1979) en la consideración de sus formas. PérezÍñigo (1984a) solo incluyó la ssp. pseudoleptocephalus Blüthgen, 1925.

\section{Lasioglossum (Evylaeus) albovirens albovirens (Pérez,} 1895)

Halictus albovirens Pérez, 1895. Esp. nouv. Mellif. Barbarie: 52

Halictus littoralis var. bequaerti Blüthgen, 1923. Arch. Naturgesch., 89A (5): 251

Subespecie íbero-magrebí, descrita de "Barbarie". Se reparte por el norte de África (en Argelia, Marruecos y en la ciudad autónoma de Melilla) y por el sur peninsular ibérico (provincias de Almería, Cádiz, Málaga y Murcia) (Blüthgen, 1923b; Ebmer, 1976a; 1979). A estas citas seguramente se refería Pérez-Íñigo (1984a).

\section{Lasioglossum (Evylaeus) albovirens belisarium} (Warncke, 1975)

Halictus leptocephalus belisarium Warncke, 1975. Vieraea, 4 (1-2) (1974): 202

Subespecie endémica de Canarias, de las islas de Gran Canaria y Tenerife.

Lasioglossum (Evylaeus) albovirens lajarense (Warncke, 1975)

Halictus leptocephalus lajarensis Warncke, 1975. Vieraea, 4 (1-2) 202(1974):

Subespecie endémica de Canarias, de las islas de Fuerteventura y Lanzarote.

\section{Lasioglossum (Evylaeus) albovirens pseudoleptoce-} phalus Blüthgen, 1925

Halictus pseudoleptocephalus Blüthgen, 1925. Arch. Naturgesch., 90A (10) (1924): 132

Subespecie atlántica, íbero-magrebí, conocida en España (La Coruña), Portugal, (Algarve: Monte Gordo y Troia) y Marruecos occidental. Pauly (2013) le asignaba estatus de especie y solo apuntaba su presencia en Portugal. Ortiz-Sánchez (2011), en su recopilación, no la incluía.

Lasioglossum (Evylaeus) alpigenum (Dalla Torre, 1877) Halictus smeathmanellus var. alpigena Dalla Torre, 1877. Z. Ferdinandeums Tirol Vorarl., 21 (3): 183
Especie europea, conocida en Alemania, Italia y Suiza, que Ortiz-Sánchez (2011) incluía en el listado de abejas de España, basándose en datos inéditos de Ebmer.

Lasioglossum (Evylaeus) angusticeps (Perkins, 1895)

Halictus angusticeps Perkins, 1895. Entomol. Mon. Mag., 31 (2) 6: 39

Halictus exetinus Cockerell, 1938. Am. Mus. Nov., 997: 4

Halictus indecisus Cockerell, 1938. Am. Mus. Nov., 997: 5

Especie paleártica occidental.

Lasioglossum (Evylaeus) arctifrons (Saunders, 1903)

Halictus arctifrons Saunders, 1903. Trans. R. Entomol. Soc. Lond., 1903: 215

Endemismo canario, representado, además de por la nominal, por las subespecies: Lasioglossum (Evylaeus) arctifrons glandarium (Warncke, 1975) y Lasioglossum (Evylaeus) arctifrons optatum (Warncke, 1975).

Lasioglossum (Evylaeus) arctifrons arctifrons (Saunders, 1903)

Halictus arctifrons Saunders, 1903. Trans. R. Entomol. Soc. Lond., 1903: 215

Subespecie endémica de las islas de La Gomera y Tenerife.

Lasioglossum (Evylaeus) arctifrons glandarium (Warncke, 1975)

Halictus arctifrons glandarium Warncke, 1975. Vieraea, 4 (12) (1974): 203 Palma.

Subespecie endémica de las islas de El Hierro y La

Lasioglossum (Evylaeus) arctifrons optatum (Warncke, 1975)

Halictus arctifrons optatum Warncke, 1975. Vieraea, 4 (1-2) (1974): 203

Subespecie endémica de la isla de Gran Canaria.

Lasioglossum (Evylaeus) articulare (Pérez, 1895)

Halictus articularis Pérez, 1895. Esp. nouv. Mellif. Barbarie: 54

Especie mediterránea, extendida desde las Islas Canarias hasta Oriente Medio. En la la Península Ibérica es escasa y en Canarias solo se conoce en la isla de Fuerteventura.

Lasioglossum (Evylaeus) aureimontanum Ebmer, 1970 Lasioglossum aureimontanum Ebmer, 1970. Naturkdl. Jb. Linz, 1970: 66

Especie mediterránea occidental. 
Lasioglossum (Evylaeus) aureolum (Pérez, 1903)

Halictus aureolus Pérez, 1903. Proc. verb. Soc. Linn. Bordeaux, 58: 211

Especie mediterránea suroccidental.

Lasioglossum (Evylaeus) brevicorne (Schenck, 1870)

Halictus brevicornis Schenck, 1870. Jahrb. Ver. Naturk. Nassau, 21/22 (1867-1868): 310

Especie paleártica occidental, con dos subespecies en el territorio estudiado.

\section{Lasioglossum (Evylaeus) brevicorne brevicorne} (Schenck, 1870)

Halictus brevicornis Schenck, 1870. Jahrb. Ver. Naturk. Nassau, 21/22 (1867-1868): 310

Halictus lucidicollis Pérez, 1895. Esp. nouv. Mellif. Barbarie: 55

Halictus analis Pérez, 1903. Proc. verb. Soc. Linn. Bordeaux, 58: 216

Halictus brevicornis aciculatus Blüthgen, 1930. En: Schmiedeknecht, Hymen. Nord-Mitteleurop.: 740

Halictus optimellus Cockerell, 1938. Am. Mus. Nov., 997: 6

Subespecie paleártica occidental, frecuente en la Península Ibérica y en Baleares.

\section{Lasioglossum (Evylaeus) brevicorne gomerense (Blüth-} gen, 1937)

Halictus brevicornis aciculatus var. gomerensis Blüthgen, 1937. Soc. Scient. Fenn., Comm. Biol., 6 (11): 2

Subespecie endémica de las Islas Canarias, conocida en la mayor parte de las islas del archipiélago: Fuerteventura, Gran Canaria, La Gomera, Lanzarote y Tenerife.

Lasioglossum (Evylaeus) buccale (Pérez, 1903)

Halictus buccalis Pérez, 1903. Proc. verb. Soc. Linn. Bordeaux, 58: 215

Especie paleártica occidental, extendida hasta Asia central.

Lasioglossum (Evylaeus) calceatum (Scopoli, 1763)

Apis calceata Scopoli, 1763. Entomol. Carniol.: 301

Hylaeus cylindricus Fabricius, 1793. Syst. Entomol., 2: 302

Melitta fulvocincta Kirby, 1802. Monogr. Apum Angliae, 2: 68

Melitta obovata Kirby, 1802. Monogr. Apum Angliae, 2: 75

Andrena vulpina Fabricius, 1804. Syst. Piezator.: 326

Halictus terebrator Walckenaer, 1817. Mém. Hist. Nat. Abeill. Solit.: 72

Hylaeus bipunctatus Schenck, 1853. Jahrb. Ver. Naturk. Nassau, 9: 160

Halictus cylindricus rhodostomus Dalla Torre, 1877. Z. Ferdinandeums Tirol Vorarl., 21 (3): 180
Halictus calceatus ulterior Cockerell, 1929. Ann. Mag. Nat. Hist., 4 (10): 588

Especie paleártica con cierta tendencia umbrófila en la Península Ibérica.

Lasioglossum (Evylaeus) capitale (Pérez, 1903)

Halictus capitalis Pérez, 1903. Proc. verb. Soc. Linn. Bordeaux, 58: 215

Halictus mauretaniae Strand, 1910. Ent. Z. Frankcf. a. M., 24: 216

Especie íbero-magrebí.

Lasioglossum (Evylaeus) castilianum (Blüthgen, 1931)

Halictus castilianum Blüthgen, 1931. Mitt. Zool. Mus. Berlin, 17: 390

Halictus hyalipennis, sensu Blüthgen, 1924 (non Morawitz, 1876)

Especie endémica de la Península Ibérica.

Lasioglossum (Evylaeus) clypeare (Schenck, 1853)

Hylaeus clypearis Schenck, 1853. Jahrb. Ver. Naturk. Nassau, 9: 162

Especie paleártica occidental, extendida hasta Irán.

Lasioglossum (Evylaeus) collopiense (Pérez, 1903)

Halictus collopiensis Pérez, 1903. Proc. verb. Soc. Linn. Bordeaux, 58: 212

Halictus soror auctt. (non Saunders, 1901)

Especie íbero-magrebí, incluidas las Islas Canarias, según Ebmer (1976 y 1988a) y Pauly (2013). Fue descrita de Argelia, citada de Canarias -como Halictus morio subsp. soror (Warncke, 1975a)-, y en España peninsular su existencia solo había sido apuntada como posibilidad (Blüthgen, 1924b): "en el sur de la península podría acaso hallarse el argelino $H$. soror Saund."). También fue señalada en el norte de África por Ebmer (1972a y 1988a) como L. morio cordiale (Pérez, 1903). Pauly (2013) desestimó esta última opinión de Ebmer, pero mantuvo la distribución de Lasioglossum collopiense en España peninsular, extremo este que, dadas las circunstancias, debería cuestionarse, a no ser que se formalicen nuevas capturas en el territorio ibérico.

\section{Lasioglossum (Evylaeus) convexiusculum (Schenck,} 1853)

Hylaeus convexiusculus Schenck, 1853. Jahrb. Ver. Naturk. Nassau, 9: 166

Halictus appropinquans Schenck, 1870. Jahrb. Ver. Naturk. Nassau, 21/22 (1867-1868): 305

Halictus porcus Morawitz, 1872. Ver. Zool.-bot. Ges. Wien., 22: 369 (partim) 
Halictus puncticollis var. vergenevensis Frey Gessner, 1903. Faun. Insect. Helvet., 1: 22

Especie paleártica occidental, extendida hasta Irán.

Lasioglossum (Evylaeus) corvinum (Morawitz, 1876)

Halictus corvinus Morawitz, $1877^{13}$. Horae Soc. entomol. Ross., 14: 91

Especie paleártica occidental, hasta el Cáucaso.

Lasioglossum (Evylaeus) crassepunctatum (Blüthgen, 1923)

Halictus crassepunctatus Blüthgen, 1923. Arch. Naturgesch., 89A (5): 280

Especie euroasiática, principalmente mediooccidental, hasta Asia central. En el territorio ibérico se conoce escasamente y solo del norte peninsular.

Lasioglossum (Evylaeus) cupromicans (Pérez, 1903)

Halictus cupromicans Pérez, 1903. Proc. verb. Soc. Linn. Bordeaux, 58: 211

Lasioglossum scoticum Ebmer, 1970. Naturkdl. Jb. Linz, 1970: 61

Lasioglossum hibernicum Ebmer, 1970. Naturkdl. Jb. Linz, 1970: 63

Especie europea occidental, con tendencia orófila, que se reparte, además de en las islas Británicas, por las montañas del sur (Alpes, Pirineos, Sierra de Guadarrama, Tatra, Balcanes y Cárpatos). Está constituida por tres o cuatro subespecies, aunque es la forma nominal la que existe en el territorio ibérico, donde aparece exclusivamente en zonas de altitud de la Sierra de Guadarrama y de Pirineos; esta subespecie se encuentra también en las islas Británicas y los Alpes occidentales.

\section{Lasioglossum (Evylaeus) dusmeti (Blüthgen, 1924)}

Halictus dusmeti Blüthgen, 1924. Mem. R. Soc. Esp. Hist. Nat., 11 (9): 387

Especie endémica de la Península Ibérica, citada, no frecuentemente, pero sí en distintos puntos a lo largo de su superficie.

\section{Lasioglossum (Evylaeus) elegans (Lepeletier, 1841)}

Halictus elegans Lepeletier, 1841. Hist. Nat. Insectes Hyménopt., 2: 286

Halictus giraudi Dalla Torre \& Friese, 1895. Ent. Nachr., 21:

38, (nomen novum para Halictus rubellus Eversmann, 1852)

Especie paleártica occidental, extendida hasta Irán.

\footnotetext{
$13 \quad$ Véase nota 11.
}

Lasioglossum (Evylaeus) euboeense (Strand, 1909)

Halictus euboeensis Strand, 1909. Arch. Naturgesch., 75, I (1): 31

Halictus furnasensis Strand, 1909. Arch. Naturgesch., 75, I (1): 35

Halictus kirschbaumi Blüthgen, 1918. Jahrb. Ver. Naturk. Nassau, 71 (1919): 214

Especie paleártica suroccidental, rara en la Península Ibérica, solo conocida en el centro, en la Sierra de Guadarrama. El registro actual es la segunda cita de esta especie en la fauna ibérica.

Nuevos Registros: España: Segovia: Sierra de Guadarrama: Venta de los Mosquitos, 1400 m, 24-IX-2009, 2 우 우, Ornosa et al. leg.; UCM. Sobre Jacobaea vulgaris Gaertn. (1754). Ebmer (1979) y Pérez-Íñigo (1984b) citaron el mismo ejemplar de una única localidad de esta misma sierra (Ávila: Arroyo del Valle de Enmedio, Peguerinos, 26-IX- 1978).

\section{Lasioglossum (Evylaeus) fratellum (Pérez, 1903)}

Halictus subfasciatus Nylander, 1848. Not. Sällsk. Fauna Flora Fenn. Förh., (Adnot.) 1: 200 (non Imhoff, 1832)

Halictus fratellus Pérez, 1903. Proc. verb. Soc. Linn. Bordeaux, 58: 214

Halictus freygessneri Alfken, 1904. Z. Syst. Hymenopt. Dipterol., 4: 3 (nomen novum para Halictus subfasciatus Nylander, 1848)

Halictus norvegicus Strand, 1910. Nyt. Mag. Naturvid., 48: 337

Halictus niger, sensu Stoeckhert, 1954 (non Viereck, 1903)

Lasioglossum nigrum, sensu Michener, 1951

Especie paleártica, con matiz bóreoalpino y marcada tendencia orófila en el sur de Europa; solo aparece en altitud y en el norte montañoso en la Península Ibérica (en la Cordillera Cantábrica y en Los Pirineos).

\section{Lasioglossum (Evylaeus) fulvicorne (Kirby, 1802)}

Melitta fulvicornis Kirby, 1802. Monogr. Apum Angliae, 2: 67 Hylaeus laeviusculus Schenck, 1853. Jahrb. Ver. Naturk. Nassau, 9: 146

Hylaeus albitarsis Schenck, 1853. Jahrb. Ver. Naturk. Nassau, 9: 148

Hylaeus bisbimaculatus Schenck, 1853. Jahrb. Ver. Naturk. Nassau, 9: 169

Halictus opacifrons Pérez, 1911. Bull. Soc. Amis Sci. Nat. Rouen, 46 (1910): 43, (nomen novum para Hylaeus fulvicornis Kirby, 1802, sensu Alfken, 1905)

Especie paleártica, con distribución disjunta, paleártica occidental, en su forma nominal por un lado y paleártica oriental, por otro.

\section{Lasioglossum (Evylaeus) glabriusculum (Morawitz,} 1872)

Halictus glabriusculus Morawitz, 1872. Verh. zool. -bot Ges. Wien., 22: 372

Halictus leucopygus Pérez, 1903. Proc. verb. Soc. Linn. Bordeaux, 58: 217 
Halictus truncatus Alfken, 1905. Cas. Csl. Spol. Ent., 2: 4 (non Robertson, 1901)

Halictus granulosus Alfken, 1906. Cas. Csl. Spol. Ent., 3: 96, (nomen novum para Hylaeus truncatus Alfken, 1905)

Especie paleártica occidental.

Lasioglossum (Evylaeus) griseolum (Morawitz, 1872)

Halictus griseolus Morawitz, 1872. Verh. zool. -bot Ges. Wien, 22: 371

Halictus labrosus Vachal, 1895. An. Soc. Esp. Hist. Nat., 24: 148 (non Say, 1837)

Halictus misellus Pérez, 1903. Proc. verb. Soc. Linn. Bordeaux, 58: 217

Halictus dubitabilis Saunders, 1904. Trans. R. Entomol. Soc. Lond., 1904: 613

Especie paleártica occidental, que llega hasta Asia central.

Lasioglossum (Evylaeus) helios Ebmer, 1985

Lasioglossum helios Ebmer, 1985. Linzer biol. Beitr., 17 (1): 206

Especie endémica de la Península Ibérica, de España. Pauly (2013) la trataba como una subespecie de Lasioglossum duckei (Alfken, 1909), especie mediterránea, extendida hasta Asia Menor.

Lasioglossum (Evylaeus) ibericum Ebmer, 1975

Lasioglossum ibericum Ebmer, 1975. Linzer biol. Beitr., 7 (1): 105

Especie ibérica, descrita de Reus (España), que se reparte por la Península Ibérica y penetra en Francia.

Lasioglossum (Evylaeus) immunitum (Vachal, 1895)

Halictus immunitus Vachal, 1895. An. Soc. Esp. Hist. Nat., 24: 148

Halictus scariosus Pérez, 1895. Esp. nouv. Mellif. Barbarie: 54

Halictus cincticornis Saunders, 1908. Trans. R. Entomol. Soc. Lond., 1908: 187

Halictus medearhensis Strand, 1909. Arch. Naturgesch.75, I (1): 41

Especie mediterránea occidental, descrita de Sevilla (España), con distribución íbero-magrebí y en Sicilia y Cerdeña.

Lasioglossum (Evylaeus) intermedium (Schenck, 1870)

Halictus intermedius Schenck, 1870. Jahrb. Ver. Naturk. Nassau, 21/22 (1867-1868): 309

Halictus melanoproctus Pérez, 1903. Proc. verb. Soc. Linn. Bordeaux, 58: 215

Halictus servulellus Strand, 1909. Arch. Naturgesch., 75, I (1): 50

Especie paleártica occidental, extendida hasta Irán.
Lasioglossum (Evylaeus) interruptum (Panzer, 1798)

Hylaeus interruptus Panzer, 1798. Faunae Insector. Ger., 55: 4 Apis basimacula Schrank, 1802. Fauna Boica, 2(2): 402

Hylaeus geminus Erichson, 1835. En: Waltl, Reise d'Tyrol, 2: 103

Hylaeus quadrimaculatus Schenck, 1853. Jahrb. Ver. Naturk. Nassau, 9: 150

Halictus opacus Pérez, 1895. Esp. nouv. Mellif. Barbarie: 54

Halictus trispinosus Alfken, 1907. Z. Syst. Hymenopt. Dipterol., 3: 202

Halictus interruptus atlanticus Cockerell, 1938. Am. Mus. Nov., 997: 3

Especie paleártica occidental, extendida hasta Irán.

Lasioglossum (Evylaeus) laetum (Brullé, 1844) ${ }^{14}$

Halictus laetus Brullé, 1844. Hist. nat. Il. Canar., 2 (Partie 2) (1836-1844): 88

Endemismo canario, ampliamente repartido por todo el archipiélago.

Lasioglossum (Evylaeus) laeve (Kirby, 1802)

Melitta laevis Kirby, 1802. Monogr. Apum Angliae, 2: 65

Hylaeus convexus Schenck, 1853. Jahrb. Ver. Naturk. Nassau, 9: 161

Halictus nigriventris Arnold, 1894. Horae Soc. entomol. Ross., 28 (1893-1894): 166

Halictus kriegeri Alfken, 1897. Ent. Nachr., 23: 104

Especie paleártica, extendida hasta Irán, con tendencia orófila en el sur de Europa.

Lasioglossum (Evylaeus) laevidorsum (Blüthgen, 1923)

Halictus laevidorsum Blüthgen, 1923. Arch. Naturgesch., 89A (5): 257

Especie mediterránea septentrional, que se extiende hasta el Cáucaso, de donde procede la cita original. De las cuatro subespecies que contiene, en la fauna ibérica, aparece la siguiente:

\section{Lasioglossum (Evylaeus) laevidorsum priesneriellum} (Warncke, 1981)

Halictus laevidorsum priesneriellus Warncke, 1981. Carinthia, 2: 171(91): 290

Subespecie mediterránea septentrional, con tendencia orófila, conocida desde el norte de España (por medio de escasos registros de Pirineos), en Francia, Suiza, Austria y Grecia.

Lasioglossum (Evylaeus) laticeps (Schenck, 1870)

Halictus laticeps Schenck, 1870. Jahrb. Ver. Naturk. Nassau, 21/22 (1867-1868): 305

14 Véase la segunda parte de la nota 3, en relación con el año de publicación de Brullé (1844). 
Hylaeus affinis Schenck, 1853. Jahrb. Ver. Naturk. Nassau, 9: 146 (non Smith, 1853)

Hylaeus nigricornis Schenck, 1853. Jahrb. Ver. Naturk. Nassau, 9: 148 (non Say, 1837)

Halictus mendax Alfken, 1912. Schr. Phys. ökon Ges. Königsb., 53: 126, (nomen novum para Hylaeus affinis Schenck, 1853)

Especie euroasiática occidental, extendida hasta Irán.

Lasioglossum (Evylaeus) leucopus (Kirby, 1802)

Melitta leucopus Kirby, 1802. Monogr. Apum Angliae, 2: 59

Especie euroasiática, con tendencia orófila en el sur, donde es abundante en cotas altas, en pinares y pastizales, de la mitad septentrional de la Península Ibérica.

Nuevos Registros: España: Segovia: Sierra de Guadarrama: Venta de los Mosquitos, 1400 m, 14-VII-2009, 1 ㅇ, Ornosa et al. leg.; UCM. Sobre Jacobaea vulgaris Gaertn. (1754). Pérez-Íñigo (1984b) la había citado de otras localidades de esta misma sierra.

Lasioglossum (Evylaeus) limbellum (Morawitz, 1876) Halictus limbellus Morawitz, 1876. En: Fedtschenko, Izv. Imp. Obsetsva. Lûbit. Estestv. Antropol. Etnogr., 21 (2): 249

Halictus limbellus dongarica Blüthgen, 1934. Konowia, 13: 159

Especie paleártica. En el área ibérica presenta la siguiente subespecie:

Lasioglossum (Evylaeus) limbellum ventrale Pérez, 1903

Halictus ventralis Pérez, 1903. Proc. verb. Soc. Linn. Bordeaux, 58: 213

Halictus gibbulus Pérez, 1903. Proc. verb. Soc. Linn. Bordeaux, 58: 214

Halictus combinatus Blüthgen, 1921. Mitt. Dtsch. Entomol.

Ges., 13: 140

Halictus rufulocinctus Cockerell, 1937. Am. Mus. Nov., 960: 7 Halictus frigescens Cockerell, 1938. Am. Mus. Nov., 997: 3

Subespecie, que de acuerdo con Ebmer (1988a) tiene distribución paleártica suroccidental, repartida hasta Europa media por el norte y hasta Malta por el este.

Lasioglossum (Evylaeus) lissonotum (Noskiewicz, 1926) Halictus lissonotus Noskiewicz, 1926. Polski Pismo Entomol., 4 (1925): 233

Especie mediterránea, orófila y rara en la Península Ibérica, donde solo se conoce, y escasamente, en la Sierra de Guadarrama.

Lasioglossum (Evylaeus) littorale (Blüthgen, 1923)

Halictus littoralis Blüthgen, 1923. Arch. Naturgesch., 89A (5): 248

Especie europea, que llega hasta la península de Crimea, por el este y hasta Alemania, por el norte. En
España existe la forma nominal en las Islas Canarias, pero en el ámbito ibérico es Lasioglossum (Evylaeus) littorale occitanicum Ebmer, 1976.

Lasioglossum (Evylaeus) littorale littorale (Blüthgen, 1923)

Halictus littoralis Blüthgen, 1923. Arch. Naturgesch., 89A (5): 248

Subespecie europea, que llega hasta las Islas Canarias.

Lasioglossum (Evylaeus) littorale occitanicum Ebmer, 1976

Lasioglossum littorale occitanicum Ebmer, 1976. Linzer biol. Beitr., 8 (1): 246

Endemismo ibérico repartido por Baleares y el centro peninsular (Ebmer, 1979).

Lasioglossum (Evylaeus) lucidulum (Schenck, 1861)

Hylaeus lucidulus Schenck, 1861. Jahrb. Ver. Naturk. Nassau, 14 (1859): 292

Hylaeus tenellus Schenck, 1861. Jahrb. Ver. Naturk. Nassau, 14 (1859): 293

Halictus gracilis Morawitz, 1865. Horae Soc. entomol. Ross., 3: 77

Halictus unguinosus Pérez, 1903. Proc. verb. Soc. Linn. Bordeaux, 58: 217

Halictus chotanensis Strand, 1909. Arch. Naturgesch., 75, I (1): 26

Especie paleártica, que llega hasta Mongolia, en el este.

Lasioglossum (Evylaeus) malachurum (Kirby, 1802)

Melitta malachura Kirby, 1802. Monogr. Apum Angliae, 2: 67

Halictus longulus Smith, 1848. Zoologist, 2: 2104

Hylaeus apicalis Schenck, 1853. Jahrb. Ver. Naturk. Nassau, 9: 161

Hylaeus coriarius Schenck, 1853. Jahrb. Ver. Naturk. Nassau, 9: 163

Halictus malachuroides Strand, 1909. Arch. Naturgesch., 75, I (1): 41

Halictus malachurus sharificus Cockerell, 1937. Am. Mus. Nov., 960: 6

Especie paleártica occidental, distribuida desde las islas Azores y Canarias (donde hay una única cita de Fuerteventura) (Hohmann et al., 1993), hasta Irán. Se reparte por el norte de África y ampliamente por Europa, desde la Península Ibérica hasta Suecia.

El hecho de que se trate de una especie eusocial, populosa, por lo tanto, resalta el que en Canarias se conozca por un solo ejemplar (una hembra de Fuerteventura) (Hohmann et al., 1993), que haría nece- 
sarios nuevos registros que confirmen su existencia en ese territorio.

Lasioglossum (Evylaeus) mandibulare (Morawitz, 1866)

Halictus mandibulare Morawitz, 1866. Horae Soc. entomol. Ross., 4: 23

Halictus carneiventris Dours, 1872. Revue Mag. Zool., 23: 311

Halictus coloratus Morawitz, 1874. Horae Soc. entomol. Ross., 10 (1873-1874): 167

Halictus czekelii Friese, 1916. Dt. Ent. Z., 1916: 30 Halictus sareptanus Blüthgen, 1923. Arch. Naturgesch., 89A (5): 290

Especie paleártica suroccidental, hasta Asia central. Ortiz-Sánchez (2011) incluía, en el territorio ibérico, además de a la subespecie nominal a la subespecie carneiventre (Dours, 1872), que Pauly (2013) estableció como una sinonimia.

Lasioglossum (Evylaeus) marginatum (Brullé, 1832)

Halictus marginatus Brullé, 1832. Expéd. Scient. Morée, 3 (1): 351

Halictus fasciatellus Schenck, 1870. Jahrb. Ver. Naturk. Nassau, 21/22 (1867-1868): 307

Halictus gribodi Kriechbaumer, 1873. Verh. Zool. -Bot. Ges. Wien., 23: 62

Halictus riparius Morawitz, 1874. Horae Soc. entomol. Ross., 10 (1873-1874): 165

Halictus vulgaris Morawitz, 1876. En: Fedtschenko, Izv. Imp. Obshch. Ljubit. Estest. Antrop. Etnog., 21: 250

Halictus kervilleanus Pérez, 1911. Bull. Soc. Amis Sci. Nat. Rouen, 46 (1910): 42

Especie paleártica, extendida hasta Nepal y hasta Marruecos, en el norte de África.

Lasioglossum (Evylaeus) maurusium (Blüthgen, 1935) Halictus maurusius Blüthgen, 1935. Dt. Ent. Z., 1935: 143

Especie mediterránea occidental, prácticamente íbero-magrebí, extendida hasta el sur de Francia.

\section{Lasioglossum (Evylaeus) mediterraneum (Blüthgen, 1926)}

Halictus mediterraneus Blüthgen, 1926. Dt. Ent. Z., 1925: 413

Especie mediterránea occidental, extendida hasta Grecia, rara y meridional en la Península Ibérica.

Lasioglossum (Evylaeus) mesosclerum (Pérez, 1903)

Halictus mesosclerus Pérez, 1903. Proc. verb. Soc. Linn. Bordeaux, 58: 210

Halictus rhodosianus Strand, 1909. Arch. Naturgesch., 75, I (1): 49

Lasioglossum balneorum Ebmer, 1974. Israel J. Ent., 9: 206
Halictus bubulcus Warncke, 1982. Bull. Mus. Civ. Stor. Nat. Venezia, 32 (1981): 88

Especie paleártica occidental, hasta Asia central.

Lasioglossum (Evylaeus) minutissimum (Kirby, 1802)

Melitta minutissima Kirby, 1802. Monogr. Apum Angliae, 2: 63

Hylaeus exilis Schenck, 1861. Jahrb. Ver. Naturk. Nassau, 14 (1859): 292

Halictus hollandi Saunders, 1904. Trans. R. Entomol. Soc. Lond., 1904: 614

Halictus kosensis Strand, 1909. Arch. Naturgesch., 75, I (1): 36

Halictus xanthosensis Strand, 1909. Arch. Naturgesch., 75, I (1): 6

Halictus costifellereus Strand, 1909. Arch. Naturgesch., 75, I (1): 29

Halictus arnoldi Saunders, 1910. Entomol. Mon. Mag., 21(2) (46): 11

Halictus lucidellus Cockerell, 1937. Am. Mus. Nov., 960: 3

Halictus lilliput Benoist, 1961. Bull. Mus. Civ. Stor. Nat. Venezia, 14: 44

Especie paleártica, distribuida desde las islas Azores y las Canarias, donde se conoce en Gran Canaria, La Gomera y Tenerife, hasta Pakistán.

Lasioglossum (Evylaeus) minutulum (Schenck, 1853)

Hylaeus minutulus Schenck, 1853. Jahrb. Ver. Naturk. Nassau, 9: 165

Hylaeus sublaevis Schenck, 1853. Jahrb. Ver. Naturk. Nassau, 9: 168

Hylaeus ambiguus Schenck, 1861. Jahrb. Ver. Naturk. Nassau, 14 (1859): 394

Halictus semipunctulatus Schenck, 1870. Jahrb. Ver. Naturk. Nassau, 21/22 (1867-1868): 308

Especie europea occidental, en la Península Ibérica es rara y solo existe en la zona nororiental.

\section{Lasioglossum (Evylaeus) morio (Fabricius, 1793)}

Hylaeus morio Fabricius, 1793. Syst. Entomol., 2: 306 Halictus morio basalis Dalla Torre, 1877. Z. Ferdinandeums Tirol Vorarl., 21 (3): 184 (non Smith, 1857)

Halictus balticus Blüthgen, 1919. Stettin. ent. Ztg., 80: 130

Especie paleártica occidental, repartida desde las islas Azores, a través del territorio ibérico, hasta el Cáucaso. En el territorio ibérico es escasa y presenta tendencia orófila.

Hohmann et al. (1993), siguiendo una comunicación personal de Ebmer, incluían en las Canarias esta especie, como Lasioglossum (Evylaeus) morio cordiale Pérez, 1903, de cuya validez dudaba Pauly (2013). La población de Canarias, según Pauly (2013) correspondería a Lasioglossum collopiense (Pérez, 1903).

Nuevos Registros: España: Segovia: Sierra de Guadarrama: Venta de los Mosquitos, 1400 m, 24-VII-2009, 
$10^{7}$, Ornosa et al. leg.; UCM. Pérez-Íñigo (1984b) la había citado en otras localidades de esta misma sierra.

Lasioglossum (Evylaeus) musculoides Ebmer, 1974

Lassioglosum musculoides Ebmer, 1974. Act. Mus. Mac. Sc. Nat:: 55

Especie endémica de la Península Ibérica, que se describió de Portugal y se conoce escasamente en España. Ascher \& Pickering (2012) incluían, además, una cita del norte de África. Pauly (2013) la consideraba como una sinonimia de Lasioglossum orihuelicum (Blüghten, 1924), si bien señalaba que sin haber examinado el tipo.

Lasioglossum (Evylaeus) nigripes (Lepeletier, 1841)

Halictus nigripes Lepetelier, 1841. Hist. Nat. Insectes Hyménopt., 2: 286

Halictus vulpinus Nylander, 1852. Not. Sällsk. Fauna Flora Fenn. Förh., (Adnot.) 2: 243 (non Andrena vulpina Fabricius, 1804)

Halictus nigripes nigroclypeatus Dalla Torre, 1877. Z. Ferdinandeums Tirol Vorarl., 21 (3): 181

Halictus nylanderi Pérez, 1903. Proc. verb. Soc. Linn. Bordeaux, 10: 214, (nomen novum para Halictus vulpinus Nylander, 1852)

Halictus syriacus Pérez, 1910. Bull. Soc. Amis Sci. Nat. Rouen, 45: 54

Especie paleártica occidental, extendida hasta Asia central. En la Península Ibérica se conoce en la mitad norte.

Lasioglossum (Evylaeus) nitidiusculum (Kirby, 1802)

Melitta nitidiuscula Kirby, 1802. Monogr. Apum Angliae, 2: 64 Hylaeus rugosulus Schenck, 1853. Jahrb. Ver. Naturk. Nassau, 9: 153 (non Pérez, 1895)

Hylaeus pusillus Schenck, 1853. Jahrb. Ver. Naturk. Nasssau, 9: 168

Halictus nitidulus Pérez, 1903. Proc. verb. Soc. Linn. Bordeaux, 58: 217 (non Prosopis nitidula Fabricius, 1804)

Halictus obsoletus Warncke, 1973. Ent. Nachr., 22: 25, (nomen novum para Halictus nitidulus Pérez, 1903)

Especie paleártica occidental. En la Península Ibérica se conoce de unas pocas localidades de la zona oriental y de la meseta, aunque con cierto carácter orófilo.

Lasioglossum (Evylaeus) nitidulum (Fabricius, 1804)

Prosopis nitidula Fabricius, 1804. Syst. Piezator.: 294

Especie paleártica occidental, extendida hasta Asia central. Presenta entre cinco y siete subespecies, varias controvertidas. En el territorio ibérico, según el caso, se incluyen una o dos.
Lasioglossum (Evylaeus) nitidulum nitidulum (Fabricius, 1804)

Prosopis nitidula Fabricius, 1804. Syst. Piezator.: 294

Halictus continentalis Blüthgen, 1944. Mitt. Dtsch. Entomol. Ges., 12: 25

Halictus smeathmanellus auctt. (non Mellitta smeathmanella Kirby, 1802)

Subespecie paleártica occidental cuya presencia en el área estudiada no está clara. Entre otros, ni Pérez-Íñigo (1984) ni Ebmer (1979; 1988a) ni Pauly (2013) la incluían en la fauna ibérica; su límite suroccidental lo fijaban en el sur de Italia. Sí lo hacía, en cambio, Ortiz-Sánchez (2011) en su recopilación.

Lasioglossum (Evylaeus) nitidulum hammi (Saunders, 1904)

Halictus hammi Saunders, 1904. Trans. R. Entomol. Soc. Lond., 1904: 615

Lasioglossum nitidulum sardinium Ebmer, 1978. Linzer biol. Beitr., 10 (1): 104

Subespecie descrita de Baleares, registrada también en Córcega y Cerdeña.

Lasioglossum (Evylaeus) orihuelicum (Blüthgen, 1924)

Halictus orihuelicus Blüthgen, 1924. Mem. R. Soc. Esp. Hist. Nat., 11 (9): 382

Lasioglossum andradei Ebmer, 1974. Naturkdl. Jb. Linz (1973): 138

Especie íbero-magrebí.

Lasioglossum (Evylaeus) parvulum (Schenck, 1853)

Melitta minuta, sensu Kirby, 1802. Monogr. Apum Angliae, 2: 61 (non Apis minuta Schrank, 1781, non Fabricius, 1798)

Hylaeus parvulus Schenck, 1853. Jahrb. Ver. Naturk. Nassau, 9: 154

Halictus striatus Schenck, 1870. Jahrh. Ver. Naturk. Nassau, 21/22 (1867-1868): 306

Halictus ferrugineipes Schenck, 1870. Jahrb. Ver. Naturk. Nassau, 21/22 (1867-1868): 306

Especie paleártica occidental, repartida desde la Península Ibérica (Ebmer, 1988), donde tiene carácter orófilo, hasta Irán. Pauly (2011b) señala su presencia en Sierra Nevada (España).

Lasioglossum (Evylaeus) pauperatum (Brullé, 1832)

Halictus pauperatus Brullé, 1832. Expéd. Scient. Morée, 3/1: 351 Hylaeus pullus Erichson, 1835. En: Waltl, Reise d'Tyrol, 2: 103 Halictus breviceps Saunders, 1879. Entomol. Mon. Mag., 15: 200

Halictus pauxillodes Strand, 1909. Arch. Naturgesch., 75, I (1): 45 
Halictus pauxillinus Cockerell, 1938. Am. Mus. Nov., 997: 6

Especie paleártica occidental.

Lasioglossum (Evylaeus) pauxillum (Schenck, 1853)

Hylaeus pauxillus Schenck, 1853. Jahrb. Ver. Naturk. Nassau, 9: 146

Hylaeus similis Schenck, 1853. Jahrb. Ver. Naturk. Nassau, 9: 146 (non Fabricius, 1793)

Hylaeus immarginatus Schenck, 1853. Jahrb. Ver. Naturk. Nasssau, 9: 148

Hylaeus flavicornis Schenck, 1853. Jahrb. Ver. Naturk. Nassau, 9: 149

Hylaeus flavicornis fuscitarsis Schenck, 1853. Jahrb. Ver. Naturk. Nasssau, 9: 149

Halictus delicatus Pérez, 1903. Proc. verb. Soc. Linn. Bordeaux, 58: 215

Halictus luteistigmatellus Strand, 1909. Arch. Naturgesch., 75, I (1): 39

Halictus pseudopunctatulus Strand, 1909. Arch. Naturgesch., 75, I (1): 48

Halictus algericolellus Strand, 1909. Arch. Naturgesch.,75, I (1): 53

Halictus schulthessi Blüthgen, 1924. En: Schulthess, Bull. Soc. Hist. Nat. Afrique du Nord, 15: 309 (non Vachal, 1903)

Especie paleártica occidental.

Lasioglossum (Evylaeus) phoenicurum (Warncke, 1975) Halictus phoenicurus Warncke, 1975. Vieraea, 4 (1-2) (1974): 210

Especie descrita de las Islas Canarias, donde se conoce en las islas de Fuerteventura y Lanzarote, y que existe además en África noratlática.

Lasioglossum (Evylaeus) podolicum (Noskiewicz, 1924) Halictus poldolicus Noskiewicz, 1924. Polski Pismo. Entomol., 3: 149

Halictus smeathmanellus petrosus Warncke, 1975. Polski Pismo Entomol., 45: 114

Halictus smeathmanellus nucleolus Warncke, 1982. Bull. Mus. Civ. Stor. Nat. Venezia, 32 (1981): 83

Especie europea suroccidental.

Lasioglossum (Evylaeus) politum (Schenck, 1853)

Hylaeus politus Schenck, 1853. Jahrb. Ver. Naturk. Nasssau, 9: 163

Hylaeus nanulus Schenck, 1853. Jahrb. Ver. Naturk. Nassau, 9: 164

Andrena pygmaea auctt. (non Fabricius, 1804)

Especie paleártica.
Lasioglossum (Evylaeus) pseudoplanulum (Blüthgen, 1924)

Lasioglossum pseudoplanulum Blüthgen, 1924. Mem. R. Soc. Esp. Hist. Nat., 11 (9): 383

Especie íbero-magrebí, incluidos el sur de Francia y la isla de Gran Canaria.

Lasioglossum (Evylaeus) punctatissimum (Schenck, 1853)

Hylaeus punctatissimus Schenck, 1853. Jahrb. Ver. Naturk. Nassau, 9: 147

Especie paleártica occidental, representada en la Península Ibérica por dos subespecies:

\section{Lasioglossum (Evylaeus) punctatissimum punctatissi-} mum (Schenck, 1853)

Hylaeus punctatissimus Schenck, 1853. Jahrb. Ver. Naturk. Nassau, 9: 147

Hylaeus flavitarsis Schenck, 1853. Jahrb. Ver. Naturk. Nassau, 9: 165

Halictus simillimus Schenck, 1870. Jahrb. Ver. Naturk. Nassau, 21/22 (1867-1868): 306

Halictus porcus Morawitz, 1872. Verh. zool. -bot. Ges. Wien, 22: 369 (partim)

Halictus grisescens Schenck, 1873. Berl. Ent. Z., 17: 259

Halictus longiceps Saunders, 1879. Entomol. Mon. Mag., 15: 200

Halictus tinitinensis ${ }^{15}$ Cockerell, 1938. Ann. Mag. Nat. Hist., 1 (11): 81

Subespecie paleártica occidental.

\section{Lasioglossum (Evylaeus) punctatissimum angustifrons} (Vachal, 1892)

Halictus angustifrons Vachal, 1892. Bull. Soc. Entomol. France, 61: 22

Halictus rubescens Pérez, 1891. Esp. nouv. Mellif. Barbarie: 55

Presenta distribución íbero-magrebí.

Ortiz-Sánchez (2011), en su recopilación, solo incluía a la forma nominal de esta especie, al igual que Pérez- Íñigo (1984a).

Lasioglossum (Evylaeus) puncticolle (Morawitz, 1872) Halictus puncticollis Morawitz, 1872. Verh. zool. -bot. Ges. Wien., 22: 370

Halictus puncticollis, sensu Saunders, 1879 (non Morawitz, 1872) Halictus saundersii Dalla Torre \& Friese, 1895. Ent. Nachr., 21: 40 (nomen novum para Halictus puncticollis, sensu Saunders, 1879)

\footnotetext{
$15 \quad$ El epíteto específico de Halictus tinitinensis Cockerell, 1938 aparece como"trinitinensis" en algunas publicaciones (Schwarz et al., 1996; Ascher \& Pickering, 2012), se trata solo de una errata.
} 
Especie paleártica occidental, extendida hasta Irán, rara en la Península Ibérica.

Lasioglossum (Evylaeus) pygmaeum (Schenck, 1853)

Hylaeus pygmaeus Schenck, 1853. Jahrb. Ver. Naturk. Nassau, 9: 165 (non Schenck, 1861)

Hylaeus nitidus Schenck, 1853. Jahrb. Ver. Naturk. Nassau, 9: 154 (non Panzer, 1798)

Halictus distinctus Schenck, 1870. Jahrb. Ver. Naturk. Nassau, 21/22 (1867-1868): 304

Especie paleártica occidental.

Lasioglossum (Evylaeus) quadrinotatulum (Schenck, 1861)

Hylaeus quadrinotatulus Schenck, 1861. Jahrb. Ver. Naturk. Nassau, 14 (1859): 393

Halictus pallipes Morawitz, 1865. Horae Soc. entomol. Ross., 3: 72

Halictus megacephalus Schenck, 1870. Jahrb. Ver. Naturk. Nassau, 21/22 (1867-1868): 308

Halictus sexsignatus Schenck, 1870. Jahrb. Ver. Naturk. Nassau, 21/22 (1867-1868): 311

Halictus dmitrijewi Morawitz, 1892. Horae Soc. entomol. Ross., 26: 146

Especie euroasiática, citada en España en la provincia de Teruel (Pauly, 2011b). Pérez-IÍñigo (1984a) situaba esta especie en el subgénero Lasioglossum.

\section{Lasioglossum (Evylaeus) quadrisignatum (Schenck,} 1853)

Hylaeus quadrisignatus Schenck, 1853. Jahrb. Ver. Naturk. Nassau, 9: 150

Hylaeus rufipes Schenck, 1853. Jahrb. Ver. Naturk. Nassau, 9: 164 (non Andrena rufipes Fabricius, 1793)

Hylaeus atratulus Schenck, 1853. Jahrb. Ver. Naturk. Nassau, 9: 165

Halictus pleuralis Morawitz, 1872. Verh. zool.-bot. Ges. Wien., 22: 371

Halictus nigerrimus Schenck, 1875. Dt. Ent. Z., 19(2): 321

Halictus commixtus Dalla Torre \& Friese, 1895. Ent. Nachr. 21: 38, (nomen novum Hylaeus rufipes Schenck, 1853)

Especie paleártica occidental, extendida hasta Irán.

Lasioglossum (Evylaeus) rufitarse (Zetterstedt, 1838)

Halictus rufitarsis Zetterstedt, 1838. Insect. Lappon., 1: 462

Halictus parumpunctatus Schenck, 1870. Jahrb. Ver. Naturk. Nassau, 21/22 (1867-1868): 306

Halictus lucidus Schenck, 1870. Jahrb. Ver. Naturk. Nassau, 21/22 (1867-1868): 309

Halictus atricornis Smith, 1870. Entomologist's Ann., 1870: 26

Especie holártica. Ebmer (1979) la citaba en Pirineos, en España, y aunque Pauly (2011b) no la incluía en el área ibérica, sí lo ha hecho recientemente.
Lasioglossum (Evylaeus) semilucens (Alfken, 1914)

Hylaeus pygmaeus Schenck, 1861. Jahrb. Ver. Naturk. Nassau, 14 (1859): 293 (non Schenck, 1853)

Halictus semilucens Alfken, 1914. Dt. Ent. Z., 1914: 281, (nomen novum para Hylaeus pygmaeus Schenck, 1861)

Especie euroasiática, extendida hasta Asia central, que en España se conoce en el centro peninsular (Pérezİ̃nigo, 1984b).

Lasioglossum (Evylaeus) setulellum (Strand, 1909)

Halictus setulellus Strand, 1909. Arch. Naturgesch., 75, I (1): 52 Hatictus foveolatus Blüthgen, 1924. Konowia, 3: 59 (non Robertson, 1902)

Especie euroasiática occidental, extendida hasta Irán.

Lasioglossum (Evylaeus) sexstrigatum (Schenck, 1870)

Halictus sexstrigatus Schenck, 1870. Jahrb. Ver. Naturk. Nassau, 21/22 (1867-1868): 310

Halictus sabulosus Warncke, 1986. Entomofauna, Suppl., 3: 126

Especie euroasiática.

Lasioglossum (Evylaeus) smeathmanellum (Kirby, 1802)

Melitta smeathmanella Kirby, 1802. Monogr. Apum Angliae, 2: 375

Especie paleártica occidental, pero solo atlántico sureuropea occidental y del norte de África.

Lasioglossum (Evylaeus) soror (Saunders, 1901)

Halictus soror Saunders, 1901. Entomol. Mon. Mag., 12 (2) (37): 209

Halictus atrovirens Pérez, 1903. Proc. verb. Soc. Linn. Bordeaux, 58: 213

Halictus atrovirens livius Warncke, 1982. Bull. Mus. Civ. Stor. Nat. Venezia, 32 (1981): 84.

Halictus morio elatus Warncke, 1975. Bull. ent. Pologne, Wroclaw, 45: 114

Especie paleártica occidental, extendida hasta Irán. Fue descrita de Mallorca, en España, pero también repartida por la mitad norte de la Península Ibérica.

Lasioglossum (Evylaeus) sphecodimorphum (Vachal, 1892)

Halictus sphecodimorphus Vachal, 1892. Bull. Soc. Entomol. France, 61: 22

Halictus coelebs Blüthgen, 1924. Mem. R. Soc. Esp. Hist. Nat., $11(9): 386$

Especie mediterránea occidental, aunque aparece también en Ucrania. Fue descrita de "Hispania" y "Barbaria", pero existe en el resto del territorio ibérico. 
Lasioglossum (Evylaeus) strictifrons (Vachal, 1895)

Halictus strictifrons Vachal, 1895. An. Soc. Esp. His. Nat., 24: 147

Especie mediterránea suroccidental, descrita de España.

\section{Lasioglossum (Evylaeus) subaenescens (Pérez, 1895)}

Halictus subanescens Pérez, 1895. Esp. nouv. Mellif. Barbarie: 54 Lasioglossum illyricum Ebmer, 1971. Naturkdl. Jb. Linz, 1971: 111

Especie paleártica occidental, extendida hasta Asia central. Su población nominal, más suroccidental, es la que se halla en la Península Ibérica, aunque es rara, solo conocida en la provincia de Madrid (Pérez-Íñigo, 1984).

Lasioglossum (Evylaeus) subhirtum (Lepeletier, 1841) Halictus subhirtus Lepetelier, 1841. Hist. Nat. Insectes Hyménopt., 2: 271

Halictus malachurops Cockerell, 1937. Am. Mus. Nov., 960: 5, (nomen novum para Halictus subhirtus auctt.) (non Lepetelier, 1841)

Especie mediterránea occidental, extendida hasta el sur de Alemania.

Lasioglossum (Evylaeus) transitorium (Schenck, 1870) Halictus transitorius Schenck, 1870. Jahrb. Ver. Naturk. Nassau, 21/22 (1867-1868): 309

Especie mediterránea. En el territorio ibérico, solo aparece una de las tres subespecies que la constituyen.

\section{Lasioglossum (Evylaeus) transitorium planulum (Pérez,} 1903)

Halictus planulus Pérez, 1903. Proc. verb. Soc. Linn. Bordeaux, 58: 216

Halictus tunicula Strand, 1909 ${ }^{16}$. Arch. Naturgesch., 75, I (1): 59

Halictus lentinicus Strand, 1921. Arch. Naturgesch., 87A (3): 273

Subespecie mediterránea occidental, con distribución rara en la Península Ibérica, repartida especialmente en altitudes medias y altas. Pérez-Íñigo (1984) la consideraba con rango específico.

Lasioglossum (Evylaeus) tricinctum (Schenck, 1874)

Halictus tricinctus Schenck, 1874. Berl. Ent. Z., 18: 161

Halictus delmasi Pérez, 1903. Proc. verb. Soc. Linn. Bordeaux, 58: 213

Especie euroasiática, repartida desde el área mediterránea occidental y Europa media hasta Asia central.

16 El intercambio del año de descripción entre Halictus tunicula Strand, 1909 y Halictus lentinicus Strand, 1921 que incluye Pauly (2011b), sin duda se trata de una errata.
Lasioglossum (Evylaeus) truncaticolle (Morawitz, 1877)

Halictus truncaticollis Morawitz, $1877^{17}$. Horae Soc. entomol. Ross., 14: 92

Halictus brevithorax Pérez, 1903. Proc. verb. Soc. Linn. Bordeaux, 58: 216

Halictus blidahensis Strand, 1909. Arch. Naturgesch., 75, I (1): 25

Halictus sudaghensis Strand, 1909. Arch. Naturgesch., 75, I (1): 55

Especie paleártica occidental, repartida, en la Península Ibérica, por la mitad norte.

Lasioglossum (Evylaeus) vergilianum (Pérez, 1903)

Halictus vergilianus Pérez, 1903. Proc. verb. Soc. Linn. Bordeaux, 58: 224

Especie endémica de la Península Ibérica.

Lasioglossum (Evylaeus) villosulum (Kirby, 1802)

Melitta villosula Kirby, 1802. Monogr. Apum Angliae, 2: 62

Melitta punctulata Kirby, 1802. Monogr. Apum Angliae, 2: 66

Halictus hirtellus Schenck, 1870. Jahrb. Ver. Naturk. Nassau, 21/22 (1867-1868): 311

Halictus medinai Vachal, 1895. An. Soc. Esp. Hist. Nat., 24: 148

Halictus pauperatulellus Strand, 1909. Arch. Naturgesch., 75, I (1): 44

Halictus barkensis Blüthgen, 1930. Mem. Soc. Entomol. Italia, 9: 224

Halictus villosulus perlautus Cockerell, 1930. Ann. Mag. nat. Hist., 1 (11): 82

Halictus rufotegularis Cockerell, 1938. Am. Mus. Nov., 997: 7 Halictus villiersi Benoist, 1941. Ann. Soc. Entomol. France, 110: 80

Especie paleártica, que llega hasta el Pacífico por Asia (Pauly, 2011b; Ascher \& Pickering, 2012), frecuente en en el territorio ibérico y presente en todas las Islas Canarias.

\section{Lasioglossum (Evylaeus) virens (Erichson, 1835)}

Hylaeus virens Erichsonn, 1835. En: Waltl, Reise d'Tyrol, 2: 102 Halictus simulans Pérez, 1895. Esp. nouv. Mellif. Barbarie: 52

Especie íbero-magrebí, conocida en la Península Ibérica, Mallorca (Baleares), y en Marruecos y Argelia, en el norte de África.

\section{Lasioglossum (Evylaeus) viride (Brullé, 1844) ${ }^{18}$}

Halictus viridis Brullé, 1844. Hist. nat. Il. Canar., 2 (Partie 2) (1836-1844): 88

Endemismo canario, representado, además de por la nominal, por las subespecies Lasioglossum (Evylaeus)

\footnotetext{
$17 \quad$ Véase nota 11.

18 Véase la segunda parte de la nota 3, en relación con el año de publicación de Brullé (1844).
} 
viride cabrerai (Warncke, 1975), Lasioglossum (Evylaeus) viride palmae Ebmer, 1993 y Lasioglossum (Evylaeus) viride unicolor (Brullé, 1844).

Lasioglossum (Evylaeus) viride cabrerai (Warncke, 1975) Halictus arctifrons cabrerai Warncke, 1975. Vieraea, 4 (1-2) (1974): 204

Subespecie endémica de Canarias, de las islas de Fuerteventura, Gran Canaria y Lanzarote.

Lasioglossum (Evylaeus) viride palmae Ebmer, 1993

Lasioglossum (Evylaeus) viride palmae Ebmer, 1993. Veröff. Übersee-Museum Bremen, 12: 776

Subespecie endémica de la isla de La Palma.

Lasioglossum (Evylaeus) viride unicolor (Brullé, 1844) ${ }^{19}$

Halictus viridis unicolor Brullé, 1844. Hist. nat. Il. Canar., 2 (Partie 2) (1836-1844): 88

Subespecie endémica de las islas de Gran Canaria y La Palma.

Lasioglossum (Evylaeus) viride viride (Brullé, 1844) ${ }^{20}$

Halictus viridis Brullé, 1844. Hist. nat. Il. Canar., 2 (Partie 2) (1836-1844): 88

Halictus alcedus Vachal, 1895. An. Soc. Esp. Hist. Nat., 24: 150

Subespecie endémica de las islas de El Hierro, La Gomera y Tenerife; en esta última, es especialmente abundante.

\section{Especies Incertae Sedis o dudosamente ibéricas}

Halictus ochraceovittatus Dours, 1872

Halictus ochraceovittatus Dours, 1872. Revue Mag. Zool., 23: 303

Descrita de Argelia, esta especie fue considerada como un sinónimo de Halictus (Halictus) fulvipes (Ebmer, 1979; Pérez-Íñigo, 1984a) y más tarde como nomen dubium (Ebmer, 1988a). Hoy en día, no obstante, aparece como una especie válida, Lasioglossum ochraceovittatus (Dours, 1872), en Fauna Europaea (Polaszek, 2004).

Halictus (Halictus) langobardicus Blüthgen, 1944

Halictus langobardicus Blüthgen, 1944. Mitt. Dtsch. Entomol. Ges., 12: 24 19, $20 \quad$ Véase la segunda parte de la nota 3, en relación con el año de
publicación de Brullé (1844).
Halictus tetrazonius var. nitens Dalla Torre, 1877. Z. Ferdinandeums Tirol Vorarl., 21 (3): 179, (nomen dubium)

Especie europea suroccidental, que aparentemente no llega a la Península Ibérica. Su inclusión por PérezÍñigo (1984a) debería confirmarse en el futuro, ya que no existen registros que la apoyen; mientras tanto ha de rechazarse su presencia en el territorio estudiado.

Halictus (Vestitohalictus) mucoreus (Eversmann, 1852) Hylaeus mucoreus Eversmann, 1852. Bull. Soc. Imp. Nat. Moscou, 25: 44

Especie euroasiática oriental, referida de la fauna española por Pérez-Íñigo (1984b) y salvo que se confirme con futuras capturas, su presencia en el territorio ibérico debe ser descartada.

\section{Halictus (Vestitohalictus) pici Pérez, 1895}

Halictus pici Pérez, 1895. Esp. nouv. Mellif. Barbarie: 53 Halictus extorris Vachal, 1902. Rev. Russ. Ent., 2: 230

Especie descrita de "Barbarie", con distribución en el norte de África y presente también en Egipto e Israel (Ebmer, 2011). La inclusión de esta especie en la fauna ibérica (Pérez-Íñigo, 1984a) deberá ser confirmada con capturas que apoyen su existencia en este territorio.

\section{Lasioglossum (Lasioglossum) quadricinctatus (Kirby,} 1802)

Especie incluida en la fauna española por PérezÍñigo (1984a), que seguramente se debe a una errata o a algún tipo de error de transcripción, ya que la especie como tal no existe.

La referencia incluida por dicho autor "Melitta quadricinctata Kirby, 1802. Monogr. Apum Angliae, 2: 79" (Pérez-Íñigo, 1984a, p. 181) es errónea, ya que en tales texto y página (Kirby, 1802) la especie descrita es Melitta quadrinotata Kirby, 1802. También es errónea la sinonimia que Pérez-Íñigo situaba a continuación, "Halictus albidulus Schenck, 1874. Berl. Ent. Z., 28: 162", ya que de acuerdo con Ebmer (1988a) es una sinonimia de Lasioglossum (Lasioglossum) prasinum (Smith, 1848).

Lasioglossum (Evylaeus) leptocephalum (Blüthgen, 1923) Halictus leptocephalus Blüthgen, 1923. Arch. Naturgesch., 89A (5): 245

Especie mediterránea suroriental, según Pauly (2013), aunque incluida en la fauna española por PérezÍñigo (1984a). Previamente, Warncke (1975) establecía las subespecies belisarius y lajarensis para sus representantes de las Islas Canarias, que Ebmer (1976 y 
Tabla 1.- Número de especies ibéricas y canarias de Halictus y Lasioglossum.

Table 1.- Iberian and Canary Halictus and Lasioglossum species.

\begin{tabular}{lcc}
\hline GÉNERO & NÚMERO DE ESPECIES & TOTAL DE ESPECIES \\
\hline Halictus (de la P. lbérica) & 33 & 126 \\
Lasioglossum (de la P. lbérica) & 93 & \\
\hline Halictus (de Canarias) & 1 & 6 \\
Lasioglossum (de Canarias) & 5 & 11 \\
\hline Halictus (de ambos territorios) & 2 & 143 \\
\hline Lasioglossum (de ambos territorios) & 9 & 143 \\
\hline
\end{tabular}

1979) asignaba a Lasioglossum (Evylaeus) albovirens (véase en este texto también). Pauly (2013) cuestionaba incluso la subespecie canaria. La presencia en la fauna ibérica, por lo tanto, deberá ser confirmada con nuevos registros.

Lasioglossum (Evylaeus) maculipes (Morawitz, 1876)

Halictus maculipes Morawitz, 1876. En: Fedtschenko, Izv. Imp. Obsetsva. Lûbit. Estestv. Antropol. Etnogr., 21 (2): 247

Especie, según Polaszek (2004), incluida en la Península Ibérica, aunque sin datos de citas concretas. Los únicos registros ibéricos existentes corresponden a Blüthgen (1924b), como "Hal. maculipes var. ventralis Pérez", aunque expresaba ciertas dudas. Autores posteriores que abordaban la fauna ibérica de halíctidos, o no la incluían en este territorio (Ebmer, 1979) o solo como sinonimia Pérez -Íñigo (1984a). Pauly (2011b) y Ascher \& Pickering (2012) le asignaban una distribución exclusivamente por Asia Central. Su presencia en la fauna ibérica debería confirmarse con nuevas capturas.

Lasioglossum (Evylaeus) marginellum (Schenck, 1853)

Hylaeus marginellus Schenck, 1853. Jahrb. Ver. Naturk. Nassau, 9: 147

Halictus marqueti Pérez, 1903. Proc. verb. Soc. Linn. Bordeaux, 58: 216

Especie europea. Su existencia en la fauna ibérica fue establecida por Blüthgen (1924) y discutida por Ebmer (1979), si bien Pérez-Íñigo (1984a) sí la mantenía y no, en cambio, Ortiz-Sánchez (2011) en su recopilación. También en este caso, su presencia en la fauna ibérica debería confirmarse con nuevas capturas.

\section{Patrón corológico}

La información relativa a la distribución geográfica se resume en las Tablas 1-4. Los dos géneros estudiados tienen distribución geográfica holártica y tropical, si bien las especies que aparecen en el territorio ibérico no llegan más allá del Reino Holártico (Tablas 2, 3 y 4), siendo, además, solo el 3,5\% de estas (2 de Halictus y 3 de Lassioglossum) las especies que alcanzan tal rango de distribución. En ambos casos, asimismo, predomina el corotipo paleártico o paleártico occidental

Tabla 2.- Patrón corológico del género Halictus de los territorios ibérico y canario.

Table 2.- Main chorotypes in the Iberian and Canary Genus Halictus.

\begin{tabular}{lcc}
\hline $\begin{array}{l}\text { DISTRIBUCIÓN } \\
\text { GEOGRÁFICA }\end{array}$ & $\begin{array}{c}\text { NÚMERO DE } \\
\text { ESPECIES }\end{array}$ & $\%$ \\
\hline Holártica & 2 & 5,55 \\
Paleártica & 11 & 30,6 \\
Paleártica occidental & 7 & 19,4 \\
Euroasiática & 2 & 5,55 \\
Europea & 0 & 0 \\
Europea occidental & 0 & 0 \\
Mediterránea & 2 & 5,55 \\
Mediterránea occidental & 3 & 8,3 \\
Endémica ibérica & 6 & 16,7 \\
Endémica balear & 2 & 5,55 \\
Endémica macaronésica & 1 & 2,8 \\
Íbero-magrebí & 0 & 0 \\
\hline
\end{tabular}


Tabla 3.- Patrón corológico del género Lasioglossum de los territorios ibérico y canario.

Table 3.- Main chorotypes in the Iberian and Canary Genus Lasioglossum.

\begin{tabular}{lcc}
\hline $\begin{array}{l}\text { DISTRIBUCIÓN } \\
\text { GEOGRÁFICA }\end{array}$ & $\begin{array}{c}\text { NÚMERO DE } \\
\text { ESPECIES }\end{array}$ & $\%$ \\
\hline Holártica & 3 & 2,8 \\
Paleártica & 14 & 13 \\
Paleártica occidental & 41 & 38 \\
Euroasiática & 9 & 8,3 \\
Europea & 2 & 1,8 \\
Europea occidental & 4 & 3,7 \\
Mediterránea & 8 & 7,4 \\
Mediterránea occidental & 10 & 9,3 \\
Endémica ibérica & 5 & 5,55 \\
Endémica balear & 0 & 0 \\
Endémica macaronésica & 5 & 4,6 \\
İbero-magrebi & 6 & 5,55 \\
\hline
\end{tabular}

(Tablas 2 y 3) y lo mismo sucede atendiendo al patrón corológico por especies (Tabla 4), donde los corotipos paleárticos en conjunto son la mayoría $(17,5 \%$ paleártico, $33,5 \%$ paleártico occidental y $7,7 \%$ euroasiáticas). Las especies mediterráneas, en cambio, y a diferencia de lo que sucede en otras familias de abejas ibéricas (Ornosa, et al., 2006, 2007 y 2008; Ortiz-Sanchez et al., 2002a y 200b), aparecen en menor proporción ( $7 \%$ mediterránea y 9,1\% mediterránea occidental) y destaca, también, una baja presencia de endemismos ibéricos $(7,7 \%)$, de especies íbero-magrebíes $(4,2 \%)$ y de endemismos macaronésicos $(4,2 \%)$ y baleáricos $(1,4 \%)$.

\section{Comentario final y conclusiones}

Es preciso apuntar que la naturaleza de un trabajo como este hace difícil extraer conclusiones distintas del propio contenido global del catálogo en sí mismo. No obstante, a modo de síntesis, puede establecerse que se han rastreado, revisado y consignado todos los taxones de Halictini de los géneros Halictus y Lasioglossum del territorio ibérico y de las Islas Canarias, sus sinonimias y citas, desde cada descripción original hasta las más recientes. Se han incluido cinco nuevas sinonimias.

El total de las especies estudiadas de Halictini de las faunas ibérica y canaria (Tabla 1) es de 143
Tabla 4.- Patrón corológico de los géneros Halictus y Lasioglossum de los territorios ibérico y canario.

Table 4.- Main chorotypes in the Iberian and Canary Genera Halictus and Lasioglossum.

\begin{tabular}{lcc}
\hline $\begin{array}{l}\text { DISTRIBUCIÓN } \\
\text { GEOGRÁFICA }\end{array}$ & $\begin{array}{c}\text { NÚMERO DE } \\
\text { ESPECIES }\end{array}$ & $\%$ \\
\hline Holártica & 5 & 3,5 \\
Paleártica & 25 & 17,5 \\
Paleártica occidental & 48 & 33,5 \\
Euroasiática & 11 & 7,7 \\
Europea & 2 & 1,4 \\
Europea occidental & 4 & 2,8 \\
Mediterránea & 10 & 7 \\
Mediterránea occidental & 13 & 9,1 \\
Endémica ibérica & 11 & 7,7 \\
Endémica balear & 2 & 1,4 \\
Endémica macaronésica & 6 & 4,2 \\
İbero-magrebí & 6 & 4,2 \\
\hline
\end{tabular}

(repartidas en 161 subespecies): 137 aparecen en el territorio ibérico, 11 de las cuales se hallan también en Canarias; 35 pertenecen al género Halictus y 102 al género Lasioglossum. En las Islas Canarias (Tabla 1) existen 17 especies, 3 de Halictus y 14 de Lasioglossum, 6 de ellas endémicas, y un total de 29 subespecies, 26 correspondientes a Lasioglossum (17 endémicas) y 3 a Halictus (1 endémica). De las 17 especies que existen en las Islas Canarias, aparte de los endemismos, 9 pertenecen a distribuciones más amplias (mediterráneas, paleárticas, etc.) y son comunes al área ibérica, y 2 no llegan al territorio peninsular, pero se extienden por el norte de África o más allá, por el entorno mediterráneo.

Se han incluido algunos nuevos registros, de los que el correspondiente a Lasioglossum euboeense constituye la segunda cita ibérica, que confirma su presencia en el centro de España, y se han desestimado como componentes ibéricos o canarios 8 especies erróneamente consideradas o bien de presencia dudosa.

En el patrón corológico (Tablas 2, 3 y 4), las especies holárticas son solo el 3,5\% de estas. En ambos géneros, asimismo, predomina el corotipo paleártico (Tablas 2 y 3) y lo mismo sucede atendiendo al patrón corológico por especies (Tabla 4), donde los corotipos paleárticos, en conjunto (paleártico, paleártico occidental y euroasiático), son la mayoría $(58,7 \%)$. Las especies mediterráneas apa- 
recen en menor proporción $(16,1 \%)$ y tienen, también, una baja presencia los endemismos ibéricos $(7,7 \%)$ y las especies íbero-magrebíes $(4,2 \%)$. El bajo número de endemismos macaronésicos $(4,2 \%)$ y baleáricos $(1,4 \%)$ también es remarcable, así como la inexistencia de endemismos baleáricos del género Halictus.

\section{Agradecimientos}

A Esther Cascales, que dio los primeros pasos en la actualización de los Halictus ibéricos. A la Dra. María Jesús Verdú, del IVIA (Valencia) y a los Dres. Isabel Izquierdo, Mercedes París, Amparo Blay y Miguel Ángel Alonso Zarazaga, del Museo Nacional de Ciencias Naturales de Madrid por permitirnos el acceso a las colecciones allí depositadas. Al Dr. Alonso Zarazaga (ICZN), además, por su inestimable ayuda en la resolución de ciertas dudas con las sinonimias de varias especies. Y, asimismo, al Dr. Pauly y a otro evaluador anónimo por sus amables sugerencias y comentarios.

\section{Referencias}

Ascher, J. S. \& Pickering, J. 2012. Discover Life bee species guide and world checklist (Hymenoptera: Apoidea: Anthophila). Última consulta: Octubre 2013. http://www.discoverlife.org $/ \mathrm{mp} / 20 \mathrm{q}$ ? guide= Apoidea_species.

Blank, S. M. \& Kraus, M., 1994. The nominal taxa described by K. Warncke and their types. (Insecta, Hymenoptera, Apoidea). Linzer biologische Beiträge, 26(2): 665-763.

Blüthgen, P., 1923a. Beiträge zur Systematik der Bienengattung Halictus Latr. (Hym.). Konowia, 2(12): $65-81$.

Blüthgen, P., 1923b. Beiträge zur Systematik der Bienengattung Halictus Latr. (Hym.). Konowia, 2(34): 123-142.

Blüthgen, P., 1923c. Beiträge zur Kenntnis der Bienengattnng Halictus Latr. Archiv fur Naturgeschichte, 89A(5): 231-332.

Blüthgen, P., 1924a. Nota sobre Halictus de Canarias y España. Boletín de la Real Sociedad Española de Historia Natural, 23: 18-19.

Blüthgen, P., 1924b. Contribución al conocimiento de las especies españolas del género Halictus. Memorias de la Real Sociedad Española de Historia Natural, 11: 331-544.

Blüthgen, P., 1925. Beiträge zur Synonymie der Bienengattung Halictus Latr. Deutsche entomologische Zeitschrift, 5: 385-419.

Blüthgen, P., 1937. Halictinae von den Kanarischen Inseln. Societas Scientiarum Fennica.Commentationes Biologicae, 6: 1-11.
Brullé, G. A., 1844. Insectes. In: P. B. Webb \& S. Berthelot. Histoire naturelle des Iles Canaries. 2 (Deuxième partie, Entomologie) (1836-1844). Mellier. Paris: 53-95.

Ceballos, G., 1956. Catálogo de los himenópteros de España. Trabajos del Instituto Español de Entomología (CSIC). Madrid. 554 pp.

Cockerell, T. D. A., 1938. Halictine bees from Morocco. American Museum Novitates, 997: 1-9.

Danforth, B. N., 1999. Phylogeny of the bee genus Lasioglossum (Hymenoptera: Halictidae) based on Mitochondrial COI sequence data. Systematic Entomology, 24(4): 377-393.

Danforth, B. N., Conway, L. \& Ji, S., 2003. Phylogeny of eusocial Lasioglossum reveals multiple losses of eusociality within a primitively eusocial clade of bees (Hymenoptera: Halictidae). Systematic Biology, 52: 23-36.

Danforth, B. N. \& Ji, S., 2001. Australian Lasioglossum + Homalictus form a monophyletic group: Resolving the "Autralian enigma". Systematic Biology, 50(2): 268-283.

Diniz, M. A., 1989. Catálogo das abelhas portuguesas. I. Ciências Biológicas Ecological Systems (Portugal), 9(1/2): 33-39.

Dikmen, F. \& Aytekin, A. M., 2011. Notes on the Halictus Latreille (Hymenoptera: Halictidae) fauna of Turkey. Turkish Journal of Zoology, 35(4): 537-550.

Dikmen, F., Radchenko, V. G. \& Aytekin, A. M., 2011. Taxonomic studies on the genus Halictus Latreille, 1804 in Turkey. Zoology in the Middle East, 54: 79100.

Dusmet, J. M., 1921. Contribución al conocimiento de los himenópteros de Portugal. Acta Congreso Oporto. Asociación Española para el Progreso de las Ciencias: 183-191.

Ebmer, A. W., 1969. Die Biene des Genus Halictus Latr. s. 1. im Grossraum von Linz I. Naturkundliches Jahrbuch der Stadt Linz, 1969: 133-183.

Ebmer, A. W., 1970. Die Biene des Genus Halictus Latr. s. 1. im Grossraum von Linz II. Naturkundliches Jahrbuch der Stadt Linz, 1970: 19-82.

Ebmer, A. W., 1971. Die Biene des Genus Halictus Latr. s. 1. im Grossraum von Linz III. Naturkundliches Jahrbuch der Stadt Linz, 1971: 63-156.

Ebmer, A. W., 1972a. Neue westpaläarktische Halictidae (Halictinae, Apoidea). Teill I. Mitteilungen aus dem Zoologischen Museum in Berlin, 48(2): 225-263.

Ebmer, A. W., 1972b. Revision der von Brullé, Lucas und Pérez beschriebenen westpaläarktischen Halictus-Arten (Halictidae, Halictinae, Apoidea), sowie Festlegung des Lectotypus von Lasioglossum (Evylaeus) angustifrons (Vachal). Polskie Pismo Entomologiczne, 42(3): 589-636.

Ebmer, A. W., 1973. Die Bienen des Genus Halictus Latr. s. l. im Grossraum von Linz (Hymenoptera 
Apoidea). Naturkundliches Jahrbuch der Stadt Linz, 1973: 123-144.

Ebmer, A. W., 1975a. Neue westpaläarktische Halictidae (Halictinae, Apoidea). Teill II-Die Gruppe des Halictus (Vestitohalictus) mucoreus (Ev.). Mitteilungen aus dem Zoologischen Museum in Berlin, 51: 161-177.

Ebmer, A. W., 1975b. Neue westpaläarktische Halictidae (Halictinae, Apoidea). Teil III. Linzer Biologische Beiträge, 7(1): 41-118.

Ebmer, A. W., 1975c. Revision der von Brullé, Lucas und Pérez beschriebenen westpaläarktischen HalictusArten (Halictidae, Halictinae, Apoidea)-Nachtrag. Polskie Pismo Entomologiczne, 45: 267-278.

Ebmer, A. W., 1976a. Halictus und Lasioglossum aus Marokko. Linzer Biologische Beiträge, 8(1): 205-266.

Ebmer, A. W., 1976b. Liste der Mitteleuropaischen Halictus und Lasioglossum Arten. Linzer Biologische Beiträge, 8(2): 393-405.

Ebmer, A. W., 1976c. Revision der von W. Nylander und J. Kriechvaumer beschriebenen Halictidae (Apoidea). Nachrichtenblatt der Bayerischen Entomologen, 25: 1-6.

Ebmer, A. W., 1978. Halictus, Lasioglossum, Rophites und Systropha aus dem Iran (Halictidae, Apoidea) sowie neue Arten aus der Paläarktis. Linzer Biologische Beiträge, 10(1): 1-109.

Ebmer, A. W., 1979. Ergänzungen zur Bienenfauna Iberiens. Die Gattungen Halictus, Lasioglossum und Dufourea (Apoidea Hymenoptera). Linzer Biologische Beiträge, 11(1): 117-146.

Ebmer, A. W., 1985. Neue westpaläarktische Halictidae V (Hymenoptera, Apoidea) sowie Festlegung von Lectotypen von Morawitz beschriebener, bisher ungeklärter Halictus-Arten. Linzer Biologische Beiträge, 17(1): 197-221.

Ebmer, A. W., 1987. Die europäischen Arten der Gattungen Halictus Latreille 1804 und Lasioglossum Curtis 1833 mit illustrierten Bestimmungstabellen (Insecta: Hymenoptera: Apoidea: Halictidae: Halictinae). 1. Allgemeiner Teil, Tabelle der Gattungen. Senckenbergiana biologica, 68(1-3): 59-148.

Ebmer, A. W., 1988a. Kritische Liste der nicht-parasitischen Halictidae Österreichs mit Berücksichtigung aller mitteleuropäischen Arten (Insecta: Hymenoptera: Apoidea: Halictidae). Linzer Biologische Beiträge, 20(2): 527-711.

Ebmer, A. W., 1988b. Die europäischen Arten der Gattungen Halictus Latreille 1804 und Lasioglossum Curtis 1833 mit illustrierten Bestimmungstabellen (Insecta: Hymenoptera: Apoidea: Halictidae: Halictinae). 2. Die Untergattung Seladonia Robertson 1918. Senckenbergiana biologica, 68(4-6): 323-375.

Ebmer, A. W., 1995. Asiatische Halictidae, 3. Die Artengruppe der Lasioglossum carinate-Evylaeus (Insecta: Hymenoptera: Apoidea: Halictidae: Halictinae). Linzer Biologische Beiträge, 27(2): 525-652.
Ebmer, A. W., 1999. Rote Liste der Biener Kärtens. Naturschutz in Kärnten, 15:239-269.

Ebmer, A. W., 2003. Hymenopterologische Notizen aus Österreich - 16 (Insecta: Hymenoptera: Apoidea). Linzer Biologische Beiträge, 35(1): 313-403.

Ebmer, A. W., 2011. Pater Andreas Werner Ebmer - ständig von Bienen begleitet. Eine autobiografische Skizze anlässlich des 70. Geburststages. Linzer biologische Beiträge, 43(2): 905-1017.

Espeso, M. A. \& Gayubo, S. F., 1988. Apidofauna de la provincia de Segovia. Acta Salmanticensia, 66: 1-81.

Gayubo, S. F., Torres, F. y Heras, C., 1990. Estudio sobre las abejas de la Sierra de Gredos (Hymenoptera, Apoidea). Cuadernos Abulenses, 11: 83-166.

Gibbs, J., 2009. Integrative taxonomy identifies new (and old) species in the Lasioglossum (Dialictus) tegulare (Robertson) species group (Hymenoptera, Halictidae). Zootaxa, 2032: 1-38.

Herrera, J., 1988. Pollination relationships in southern Spanish Mediterranean shrublands. Journal of ecology, 76: 274-287.

Hohmann, H., La Roche, F., Ortega, G. \& Barquin, J. 1993. Bienen, Wespen und Ameisen der Kanarischen Inseln (Insecta: Hymenoptera: Aculeata) I. Veroeffentlichungen aus dem Uebersee-Museum Bremen Naturwissenschaften, 12(I-III): 14-712.

Kerzhner, J. M., 1984. Dates of publications of "Trudy Russkogo Entomologischeskogo Obshchestva" and "Hoare Societatis Entomologicae Rossicae", 18611932. Revue d'Entomologie de l'Urss, 63(4): 1984.

Kirby, W., 1802. Monographia Apum Angliae. II. J. Raw. Ipswich. $388 \mathrm{pp}$.

La Greca, M. 1990. The Insect Biogeography of West Mediterranean Islands. International Symposium on Biogeographical aspects of insularity. Accademia Nazionale dei Lincei. Roma: 469-491.

Louadi, K. 1999. Contribution à la connaissance des genres Halictus et Lasioglossum de la región de Constantine (Algérie) (Hymenoptera, Apoidea, Halictidae). Bulletin de la Société entomologique de France, 104(2): 141-144.

Michener, C. D., 1974. The Social Behaviour of the Bees. Harvard University Press. Cambridge. 404 pp.

Michener, C. D., 2007. The bees of the world. 2nd edition. The Johns Hopkins University Press. Baltimore and London. viii + 913 pp.

Morawitz, F., 1879. Nachtrag zur Bienenfauna Caucasiens. Horae Societatis entomologicae Rossicae, [18771879], 14: 3-112.

Ornosa, C., 2001. Anotaciones relativas a ciertas publicaciones antiguas sobre apoideos (Hymenoptera, Apoidea). Boletín de la Asociación española de Entomología, 25(3-4): 17-30.

Ornosa, C., Ortiz-Sánchez, F. J. \& Torres, F., 2007. Catálogo de los Megachilidae del Mediterráneo occi- 
dental (Hymenoptera, Apoidea). II. Lithurgini y Megachilini. Graellsia, 63(1): 111-134.

Ornosa, C., Ortiz-Sánchez, F. J. \& Torres, F., 2008. Catálogo de los Megachilidae del Mediterráneo occidental (Hymenoptera: Apoidea) III. Anthidiini y Dioxyini. Graellsia, 64(1): 61-86.

Ornosa, C., Torres, F. \& Ortiz-Sánchez, F.J. 2006. Catálogo de los Megachilidae del Mediterráneo occidental (Hymenoptera, Apoidea). I. Osmiini. Graellsia, 62(2): 223-260.

Ortiz-Sánchez, F. J., 2006. Lista preliminar de los Apoidea (Hymenoptera) de Andalucía (Sur de la Península Ibérica). Acta granatense, 4/53: 22-39.

Ortiz-Sánchez, F. J., 2011. Lista actualizada de las especies de abejas de España (Hymenoptera: Apoidea: Apiformes). Boletín de la Sociedad Entomológica Aragonesa, 49: 265-281.

Ortiz-Sánchez, F. J., Ornosa, C \& Dathe, H. H., 2002a. Catálogo sinonímico de los Colletidae ibéricos. I, subfamilia Hylaeinae (Hymenoptera, Apoidea). Entomofauna, 23(21): 249-266.

Ortiz-Sánchez, F. J., Ornosa, C. \& Kuhlmann, M., 2002 b. Catálogo sinonímico de los Colletidae ibéricos. II, subfamilia Colletinae (Hymenoptera, Apoidea). Entomofauna, 23(22): 267-278.

Pagliano, G., 1988. Catalogo degli Imenotteri italiani I. Halictidae. Bollettino del Museo Civico di Storia Naturale di Venezia, 38: 85-128.

Parker, L., 1991. The evolution of social behaviour and nest architecture in sweat bees of subgenus Evylaeus. A phylogenetic approach. Behavioral ecology and sociobiology, 29(3): 153-160.

Patiny, S. \& Michez, D., 2006. Phylogenetic analysis of the Systropha Illiger 1806 (Hymenoptera: Apoidea: Halictidae) and description of a new subgenus. Annales de la Société entomologique de France (n.s), 42(1): 27-44.

Pauly, A., 2011a. Atlas Hymenoptera: Genus Seladonia. Ultima consulta: Febrero 2013. http://www.zoologie.umh.ac.be/hymenoptera/page.asp?ID=67.

Pauly, A., 2011b. Atlas Hymenoptera: Evylaeus sensu Ebmer, excepté les espèces à reflets métalliques (voir Dialictus). Última consulta: Octubre 2013. http://www. atlashymenoptera.net/page.asp?id=95.

Pauly, A., 2011c. Atlas Hymenoptera: Vestitohalictus. Ultima consulta: Febrero 2013. http://www.atlashymenoptera.net/page. asp?id $=97$.

Pauly, A., 2011d. Espèces du sous-genre Lasioglossum d'Europe et du Bassin Méditerranéen. Última consulta: Octubre 2013. http://www.atlashymenoptera. net/page. asp?id $=105$.

Pauly, A., 2011e. Atlas of the European Bees: genus Lasioglossum, subgenus Lasioglossum. STEP Project, Atlas Hymenoptera, Mons, Gembloux. Última consulta: Octubre 2013. http://www.zoologie.umh.ac.be/hymenoptera/page.asp?ID=200.
Pauly, A., 2013. Dialictus de la Région Paléarctique. Ultima consulta: Octubre 2013. http://zoologie.umh. ac.be/hymenoptera/page.asp?id=128.

Pauly, A., Pesenko, Yu \& Radchenko, V., 2011. Les Halictus sensu stricto d'Europe et du Bassin Méditerranéen. Última consulta: Octubre 2013. http://www.atlashymenoptera.net/page.asp?id=70.

Pérez, J., 1895a. Espèces nouvelles de Mellifères de Barbarie (Diagnoses préliminaires). Gounouilhou. Bordeaux. 64 pp.

Pérez, J., 1895b. Voyage de M. Ch. Allaud aux Illes Canaries (novembre 1889 - juin 1890). $4^{\mathrm{e}}$ mémoire : Hymenoptères. Annales de la société entomologique de France, 44: 191-204.

Pérez, J., 1903. Espéces nouvelles de Melliféres paléarctiques. Procès-Verbaux de la Société Linnéenne de Bordeaux, 58: 208-236.

Pérez-Íñigo, C., 1984a. Lista sinonímica de las especies españolas de los géneros Halictus y Lasioglossum. Miscellania Zoologica, 8: 177-188.

Pérez-Íñigo, C., 1984b. Los ápidos de la Sierra de Guadarrama III. Familias Colletidae y Halictidae. Graellsia, 40: 129-157.

Pesenko, Y. A., 1984a. Synonymic annotated catalogue of species group names of the bees of the genus Halictus Latreille sensu stricto in the world fauna. Trudy Zoologicheskogo Instituta, 128: 16-32.

Pesenko, Y. A., 1984b. A subgeneric classification of bees of the genus Halictus Latreille sensu stricto. Entomologicheskoe Obozrente, 63(2): 340-357.

Pesenko, Y. A., 1984c. Systematics of the bees of the genus Halictus Latreille with a description of the 7th and 8th metasomal sterna of males. Trudy Zoologicheskogo Instituta, 128: 33-48.

Pesenko, Y. A., 2000. Phylogeny and classification of the family Halictidae revised (Hymenoptera: Apoidea). Journal of the Kansas Entomological Society, [1999], 72(1): 104-123.

Pesenko, Y. A., 2004. The phylogeny and classification of the tribe Halictini, with special reference to the Halictus genus-group (Hymenoptera: Halictidae). Zoosystematica Rossica, 13: 83-113.

Pesenko, Y. A., 2005. New data on the taxonomy and distribution of the Palearctic halictids: Genus Halictus Latreille (Hymenoptera: Halictidae). Entomofauna, 26 (18): 313-348.

Pesenko, Y. A., 2007. Subgeneric classification of the Paleartic bees of the genus Evylaeus Robertson (Hymenoptera: Halictidae). Zootaxa, 1500: 1-54.

Polaszek, A., 2004. Fauna Europaea: Apidae. In: Fauna Europaea: Hymenoptera: Apocrita. Fauna Europaea version 1.1 (ed. J. Noyes), http://www.faunaeur.org. (last updated 23 July 2012). Última consulta: Octubre 2013.

Schwarz, M., Gusenleitner, F., Westrich, P. \& Dathe, H. H., 1996. Katalog der Bienen Österreichs, 
Deutschlands und der Schweiz (Hymenoptera, Apidae). Entomofauna, suppl. 8: 1-398.

Schwarz, M. P., Richards, M. H. \& Danforth, B. N., 2007. Changing paradigms in Insect Social Evolution: Insights from Halictinae and Allodapine Bees. Annual Review of Entomology, 52: 127-150.

Straka, J., Bogusch, P. \& Pridal, A., 2007. Apoidea: Apiformes (Véely). Acta Entomológica Musei Nationalis Pragae. Supplementum, 11: 241-299.

Suárez, F. J. \& Martínez, J., 1972. Ápidos de la provincia de Almería. $1^{\text {a }}$ Nota. (Hymenoptera, Apoidea). Archivos del Instituto de Aclimatación, 17: 5-20.

Vachal, J., 1895. Halictus noveaux de la collection Medina. Anales de la Real Sociedad Española de Historia Natural, 24: 147-150.

Vigna Taglianti, A., Audisio, P., Biondi, M., Bologna, M.A., Carpaneto, G.M., De Biase, A., Fattorini, S., Piattella, E., Sindaco, R., Venchi, A., \& Zapparoli, M., 1999. A proposal for a chorotype classification of the Near East fauna, in the framework of the Western Palearctic region. Biogeographia, 20: 31-59.

Wcislo, W. T. \& Danforth, B. N., 2007. Solitarios en segunda instancia: Pérdida evolutiva del comportamiento social. In: E. G. Leight Jr., E. A. Herre, J. B. C. Jackson y F. Santos-Granero (Eds). Ecología y Evolución en los trópicos. Smithsonian Tropical Research Institute. Nova Art. Panama: 259-268.

Warncke, K., 1975a. Zur kenntis der bienengauttung Halictus Latr. auf den Kanarischen Inseln (Hym., Apoidea). Vieraea, 4(1-2): 201-223.

Warncke, K., 1975b. Beitrag zur Systematik und Verbreitung der Furchenbienen in der Türkei (Hymenoptera, Apoidea, Halictus). Polskie Pismo Entomologiczne, 45: 81-128.
Warncke, K., 1981. Die Bienen des Klagenfurter Beckens (Hymenoptera, Apidae). Carinthia II, 171(91): 275348.

Warncke, K., 1984. Ergänzungen zur verbreitung der bienengattung Halictus Latr. in der Türkei (Hymenoptera Apidae). Linzer Biologische Beiträge, 16 (2): 277-318.

Webb, P. B. \& Berthelot, S. 1844. Histoire naturelle des Iles Canaries. Tome Deuxième (Deuxième partie, Contenant la Zoologie) (1836-1844). Mellier. Paris. 